Lorsque $t_{1}$ et $t_{q}$ restent constants, le produit $p_{1} l$ a une valeur constante bien définie et l'on a :

$$
\frac{W}{p_{1} l}=\text { constante. }
$$

Si la matière qui entoure le premier cylindre peut ellemême être assimilée à un second cylindre de rayon $p_{2}$ et dont. la surface externe serait à une température $t_{3}$, on pourra ecrire :

$$
Q=\frac{2 \pi l K_{1}\left(t_{2}-t_{3}\right)}{\log \cdot n \cdot \frac{p_{2}}{P_{1}}}
$$

$K_{1}$ représentant le coefficient de conductibilité de la matière qui compose ce second cylindre.

Si ce second cylindre est lui-même entouré d'une matière de conductibilité $K^{\prime}$, la quantité de chaleur qu'il dégage sera, pour une surface virtuelle ayant une température $t_{s}$ et un rayon $p_{3}$ :

$$
Q^{\prime}=\frac{2 \pi l K^{\prime}\left(t_{3}-t_{4}\right)}{\log \cdot n \cdot \frac{P_{3}}{P_{2}}}
$$

Or si l'on pose $\mathrm{P}_{3}-\mathrm{P}_{2}=\lambda$ et si $\lambda$ est très petit, log.n. $\frac{f_{3}}{\hat{p}_{2}}$ peut se remplacer approximativement par $\frac{\lambda}{P_{2}}$, de sorte que :

$$
Q^{\prime}=2 \pi l K^{\prime}\left(t_{3}-t_{4}\right) \frac{\rho_{2}}{\lambda}
$$

Fours à graphite. - Dans cette sorte de four's on doit surtout s'attacher à atteindre la température la plus élevée possible. Pour y arriver on dispose dans l'axe du four, et d'une extrémité à l'autre de celui-ci, une série de lames de charbon que l'on noie dans une masse composée de charbon, d'anthracite ou de coke. L'anthracite, qui est le plus généralement employé, est un mauvais conducteur de l'électricité ; aussi, au début de l'opération, le courant passe-t-il à peu près exclusivement à travers les lames de charbon; mais, grâce au carbone qui se dégage de ces lames, l'anthracite se transforme peu à peu en graphite et devient conducteur de l'électricité. Ce type de four peut être rangé à la fois dans les deux catégories de fours à résis. tances.

La chaleur dégagée dans ces fours produit plusieurs effets : elle élève la température des matières à traiter, elle volatilise les corps autres que le charbon, enfin elle favorise less réactions chimiques. Les pertes par radiation sont déterminées par l'équation (5) et l'on voit qu'elles sont d'autant plus élevées que les divers termes du second membre sont plus grands.

-Fours à carborundum. - La charge d'un four est formèe de sable en poudre et de coke; à l'intérieur on dispose un conducteur central constitué soit par du charbon granulé, soit par des lames de charbon réunies entre elles à l'entrée et à la sortie du four. Le passage du courant dans le conducteur central dégage une chaleur considérable qui provoque la combinaison du carbone et de la silice; mais on est obligé de régler la température, car si elle venait à dépasser certaines limites, le carborundum se redécomposefait en ses éléments avec volatilisation de la silice.

"Lors de la combinaison du carbone et de la silice, il se forme, à la périphérie de la zone de production un léger dépôt d'une matière amorphe appelée par les Américains, " white stuff» (matière blanche). Cette poudre est:ôn produit intermédiaire dont les températures de formation ot de décomposition sont très rapprochées l'une de l'autre, condition qui rend sa fabrication, ou celles dautres produits similaires, des plus délicates. Aussi Acheson a cherché à régulariser la température et il y est arrivé en employant plusieurs conducteurs centraux, séparés en général les uns des autres, mais réunis en certains points déterminés par les conditions de travail du four.

Les dimensions du conducteur central sont données par les équations (3) et (4); dans cette dernière $f_{1}$ représente alors à la fois le rayon du conducteur central et celui de la surface interne du carborundum à la température $t_{\text {a }}$ étant le rayon et $t_{3}$ la température de la surface externe du carborundum.

Afin de réaliser la production maximum en carborundum il faut que $K$, conductibilité de la masse cristalline, soit le plus grand possible, c'est-à-dire que le mélange qui entoure le conducteur central ait la plus grande densité possible. $\left(t_{2}-t_{3}\right)$ devra aussi être fait aussi grand qu'on lo pourra; mais $t_{3}$ est déterminée, c'est la température qui est immédiatement inférieure à celle de formation du carborundum, et $t_{2}$ ne peut croître au delà de certaines limites carle carborundum se décomposerait.

Pour diminuer les pertes par rayonnement on doit faire $K^{\prime}$ et $\left(t_{3}-t_{4}\right)$ de la formule (5) aussi petit que possible. 0n ne peut pas modifier $K^{\prime}$, car la surface externe du carbo. rundum doit être composée du mélange de coke et de sable, matières constituant le produit que l'on veut obtenir; toutefois en donnant une épaisseur convenable à ce mélange on peut réduire $\left(t_{3}-t_{4}\right)$.

On remarquera que $p_{\mathrm{q}}$ croît au fur et à mesure de la pro. duction du carborundum; il en résulte qu'il arrive un moment où $Q^{\prime}=Q$, c'est-à-dire un moment à partir duquel il ne se produit plus de carborundum puisque toute la chaleur fournie par l'énergie électrique est alors absorbée par le rayonnement.

Il est évident que pour obtenir des résultats économiques, i) faut arrêter lopération bien avant d'atteindre ce point.

(Extrait des comptes rendus de la quatrième assemblee de la Societé Electrochimique AmériCAine).

\section{A propos de la meilleure utilisation des Chutes}

En écrivant mon article «Transport de Force et Electro. chimie » $\left(^{\star}\right)$ je n'ai voulu faire et n'ai fait que l'esquisse d'un sujet vaste et compliqué entre tous; mais comme il a trait aux problèmes économiques du plus haut intérêt en matière de houille blanche, ce sujet m'a valu de la part de nos lec. teurs un grand nombre d'observations touchant les propositions formulées dans l'article en question. J'ai ainsi pu constater que si tous les chutards sont d'accord sur ce que j'ai appelé le principe de la meilleure utilisation des chutes, certains contestent cependant les déductions auxquelles conduit la mise en pratique de ce principe. Ces divergences de vue proviennent beaucoup plus, je crois, du fait de mon exposé trop succinct que d'une véritable opposition de théo. ries. A vouloir condenser en quelques pages un sujet dont le développement comporterait un volume, l'auteur s'ex" pose à des objections qui le mettent, semble-t-il, en contradiction avec les idées reçues par la majorité des lecteurs auxquels il s'adresse. Ayant donné sur cet écueil, je veux aujourd'hui essayer de m'en dégager. Mais, soit dit en pas. sant, je ne regrette en aucune façon d'avoir provoqué cette discussion sur le problème posé:n'est-ce pas dans l'échange des idées qu'on trouve les solutions pratiques. Vous avouerais-je même que je l'ai un peu faịt exprès !...

(*) Voir numéro I I (novembre rgo3), page 353 et suivantes. 
Pour faciliter la lecture de cette suite ä món précédent article, je vais d'abord, en quelques mots, résumer son thème - qu'on a très improprement et en me faisant trop d'honneur qualifié de théorie, car je n'ai rien dit qui ne fût déjà bien connu;- - cela fait, je compléterai par une discussion plus approfondie les points qui ont donné lieu aux objections, et je terminerai par l'étude de dispositifs hydroélectriques nouveaux, susceptibles d'emploi dans certaines usines où ils permettraient de toujours réaliser l'application du principe de la meilleure utilisation.

On m'objectera peut-être que j'ai le tort d'émettre ici des idées, au lieu d'indiquer des faits, de proposer des inventions au lieu de décrire des appareils ayant fait leur preuve, mais - qu'on me permette cette parenthèse - j'estime que le rôle d'une Revue technique n'est pas seulement de faire connaitre les nouveautés industrielles, c'est-à-dire les travaux d'art, les machines, les appareils, les découvertes produits au jour le jour, mais encore de répandre dans le monde des travailleurs des conceptions susceptibles d'engendrer un progrès: Un article purement descriptif apporte à Phydraulicien, au chimiste ou à l'électricien, des renseignements sur la solution plus ou moins ingénieuse de tel'ou tel problème professionnel et quelquefois lui suggère des solutions plus heureuses, mais rarement cet article l'amène à réfléchir sur des idées neuves, précisément parce qu'on en écarte avec soin toute idée qui n'a pas encore reçu la consécration de la pratique. Il peut être bon cependant d'aller parfois de l'avant par l'imagination, même en fait d'installations hydro-électriques; une simple conception émanée de la plume du théoricien peut mettre les praticiens sur la voie d'applications fécondes en résultats matériels. C'est pourquoi je fais passer ici un grand nombre d'articles (et en particulier celui-ci) conçus dans cet esprit.

\section{ÉNONCÉ ET DISCUSSION DU PROBLÈME}

Résumé du précédent article. - Me plaçant sur le terrain del'économie politique, j'ai posé cette question: Quel objectif doit viser l'ingénieur dans l'aménagement des forces hydrauliques pour en obtenir le maximum de rendement?

J'ai essayé d'y répondre en me basant sur cette considé. ration, qu'étant donné, suivant les saisons, le débit très variable des rivières où il est possible d'aménager des chutes, le problème comporte deux termes: ou bien obtenir en chaque usine une puissance sensiblement constante, quelles que soient les variations de débit; ou bien créer une puissance qui, avec le débit, varie dans des limites telles que les variations puissent être constamment utilisées.

Dès lors, deux solutions différentes apparaissent suivant que le cours d'eau est ou n'est pas susceptible de régularisation par un lac ou un réservoir artificiel. - Dans le premiër cas, connaissant la superficie du réservoir, les graphiques de débit de la rivière nous indiquent de combien nous devons relever le niveau pour, en emmagasinant à l'époque des hautes eaux une partie du débit qui sera restitué à la chute au moment des eaux basses, obtenir le maximum de puissance à peu près constante. D'autre part, ce réservoir fait, à chaque instant, office d'accumulateur qui rend à la chute, aux heures de travail, le tébit non absorbé lors des périodes d'arrêt ou de faible activité. Il s'en suit que quel que soit le mode d'utilisation - continu ou intermittent du travail de l'eau qu'on se propose, on obtient dans ce cas la meilleure utilisation possible de l'énergie de la rivière. Mais cette solution est loin d'être générale et il faut surtout considérer le cas du cours d'eau non susceptible de régularisation.

Sur une rivière à flux non régularisable, on peut, ai-je dit, considérer la puissance d'une chute comme la somme de deux quantités : l'une constante dont la valeur est déterminée par le débit minimum minimorum ; l'autre pariable dont la valeur est, à chaque époque de l'année, fonction du débit dérivé en sus du minimum. Dans le but de simplifier le langage, tout en employant des expressions qui désignent sans ambiguité possible la fonction caractéristique de chacune déces quantités, j'ai appelé chenaux permanents ceux dont se compose la partie constante de la puissance, et chevaux. périodiques ceux, revenant à période à peu près fixe dans l'année, dont se compose la partie variable. Et j'ai écrit que la puissance totale ou puissance industrielle d'une chute est égale à la somme des chevaux permanents et des chevaux périodiques.

Cela posé, je me suis demandé quelle devait être, suivant la nature des rivières sur lesquelles on se propose d'aménager des chutes, l'importance des chevaux périodiques par rapport aux chevaux permanents, autrement dit dans quelles circonstances on avait intérêt à aménager les premiers seuls ou avec une plus ou moins grande quantité des seconds. Tout le problème de la meilleure utilisation est là.

Une règle déduite de l'étude des installations existantes, assez générale et comportant très peu d'exceptions, nous permet de résoudre cette question avec une suffisante approximation, c'est la suivante : Le cô̂t d'établissement du cheval et, par suite, son prix de revient annuel sur l'arbre des turbines (point essentiel à considérer) est en raison directe du débit et en raison inverse de la hauteur. de chute. J'ai appliqué cette règle au cas qui nous occupe en envisageant séparément : $\mathrm{I}^{\circ}$ le coût d'installation $\mathrm{du}$ cheval permanent et $2^{\circ}$ celui du cheval périodique. Elle nous montre que :

Dans une chute à gros. débit $\left(50^{\mathrm{m}^{3}}\right.$ par exemple) et à faible hauteur ( 5 mètres) les frais d'aménagement du cheval périodique sunt très élevés et de l'ordre de grandeur du prix du cheval permanent, puisqu'ils s'évaluent en raison directe du débit à dériver en sus du minimum, débit qui doit être important si l'on veut un accroissement sensible de puissance. Quand, au contraire, il s'agit d'une chute à faible débit ( $3 \mathrm{~m}^{3}$ par exemple) et à grande hauteur. (200 mètres), ces frais d'installation du cheval périodique deviennent presque négligeables devant le coût du cheval permanent, puisqu'un petit débit dérivé en sus du minimum coûte fort peu d'aménagement en donnant une très sensible augmentation de puissance.

Les chevaux périodiques sont de par leur variabilité même d'une affectation peu commode; ils ne peuvent pas être employés dans un transport d'énergie car leur périodicité ne concorde que par extraordinaire avec les variations de puissance consommée par un service public; seule une industrie privée dont les opérations peuvent être en synchronisme avec la périodicité de ces chevaux est suscep. 
tible de les absorber. Il va sans dire que nous ne nous occupons ici que des installations dont la puissance industrielle est supérieure à $\mathrm{I} 000 \mathrm{chx}$, c'est-à-dire de celles qui seront de beaucoup les plus nombreuses dans l'avenir, l'évolution économique de la houille blanche devant amener l'utilisation des rivières par des usines de plus en plus puissantes, accroître la densité d'énergie par chute. Or, en de telles installations on sera fréquemment conduit à aménager de 500 à $10000 \mathrm{chx}$ périodiques. A quoiles employer? Dans les vallées des pays de houille blanche les industries mécaniques susceptibles d'absorber beaucoup de force à dates fixes sont rares et on ne peut escompter leur développement pour l'utilisation de l'énorme quantitć de chevaux périodiques en puissance dans nos rivières aménageables. Il n'y a que les industries électrochimiques et électrométallurgiques qui soient capables de les employer. Mais n'oublions pas que pour appliquer avec bénéfice la forme électrique de l'énergie aux opérations de la métallurgie et de la grande industrie chimique, il faut que le prix de revient de cette énergie soit très bas. Les chevaux périodiques des moyennes et hautes chutes sont dès lors les seuls à pouvorr satisfaire à cette condition.

De ces considérations il résulte que dans les basses chutes on n'a pas, en général, avantage à installer des chevaux périodiques, mais que par contre on commettrait une lourde faute technique en ne les aménageant pas dans les hautes chutes, qu'on en ait ou non l'utilisation immédiate.

\section{D'oủ j'ai été conduit à écrire cette conclusion :}

Les basses chutes, celles où le cheval permanent coûte cher, né peuvent logiquement servir qu'à l'alimentation des services publics par transport d'énergie pour éclairage, force ou traction. Cette circonstance, ajoutai-je, est d'ailleurs en parfait accord avec leur position géographique; elles se trouvent dans les vallées larges, régions des centres industriels et de plus dense population où le kilowatt, produit et vendu à meilleur marché que l'énergie de la houille noire, profite à l'intérêt général tout en assurant la rémunération de l'entreprise. Au contraire, les hautes chutes sont en situation de trouver deux emplois de leur puissance : l'alimentation d'un service public avec les chevaux permanents, et la fourniture du courant à des usines électrochimiques avec les chevaux périodiques. Comme les premiers sont d'une part les plus coûteux de production, et que d'autre part ils doivent être transportés onéreusement assez loin pour atteindre les centres de consommations rares dans les vallées de grande altitude, les usines génératrices ne font ordinairement pas sur eux un plus grand bénéfice que les basses chutes. Mais les chevaux périodiques, étant presque gratuits et utilisés sur place, peuvent entrer dans la fabrication de produits électrochimiques à des prix rémunérateurs et tels que cette jeune industrie supplantera certainement les anciens procédés de la chimie et de la métallurgie. La meilleure utilisation est donc pour les moyennes et hautes chutes de faire à la fois du transport de force et de l'électrochimie, tandis qu'elle est de ne faire que du transport d'énergie pour les basses chutes.
Discussion des principales objections soulevées. $-\mathrm{Ce}_{e}$ raisonnement a, comme je l'ai dit en commençant, provoqué de nombreuseś observations dont voici les principales dans leur ordre méthodique. Sans avoir la prétentiont d'y faire face avec une suffisante logique, j'espère néanmoins démontrer qu'aucune des objections qu'on peut faire, touchant la solution préconisée de ce problème de la meilleure utilisation, ne porte sur des résultats incompatibles.

La détermination à peu près exacte et du nombre, et de la périodicité, et du prix de revient des chevaux périodiques, détermination sur laquelle repose d'une manière fonda. mentale toute application de votre " théorie suppose, m'a-t-on objecté, la connaissance parfaite du régime de la rivière. "On déterminera, dites-vous, au moyen de la courbe de régime, et par un devis d'avant-projet, le chiffre du débit qui donne le meilleur prix de revient pour le cheval périodique ». Mais cette courbe de régime pour combien de rivières peut-on actuellement l'établir? Si dans les installations existantes les industriels ont $\mathrm{pu}, \dot{a}$ l'aide de leurs barrages ou de leurs canaux, jauger les débits qu'ils emploient, remarquez que la détermination précitée ne s'est faite qu'après coup, c'est-à-dire une fois ces ouvrages en service et par conséquent trop tard pour savoir s'ils répondaient d'une façon satisfaisante à ce but de la meilleure utilisation. Il faudrait donc posséder, avant toute utilisation, les graphiques de débit de la rivière; et comme on ne les a pas, on ne peut appliquer votre " théorie" faute de cette base; il n'y a qu'à procéder par à-peu-près.

Cette objection est assurément de nature à embarrasser l'installateur de chutes. - Je l'avais néanmoins prévue: "Malheureusement, disais-je, l'incertitude inhérente à l'une des données du problème nous empêche d'opérer d'une manière aussi précise que je l'ai admis. Le profil en long de la dérivation nous fournit avec exactitude la hauteur de chute et une prévision suffisamment approchée des frais d'installation. Ce qui nous manque, c'est la connaissance des débits, et tant que nous ne pourrons pas construire les graphiques de régime, nous en serons réduits à procéder par à-peu-près ». Mais cette indétermination ne sera pas perpétuelle. On sait que le Ministre de l'Agriculture a, l'année dernière, chargé une mission de faire l'étude des grandes forces hydrauliques. MM. René TAvernier et DE LA Brosse, ingénieurs en chef des Ponts et Chaussées, à qui çette mission a été confiée, procèdent aujourd'hui au jaugeage méthodique et précis de nos rivières susceptibles d'utilisation industrielle. C'est là un travail de très longue haleine dont nous derons attendre patiemment les résultats. 11 ne pouvait être entrepris par des ingénieurs plus compétents ni plus dévoués à la cause qui nous occupe et nous pouvons être sûrs, étant.donné le soin et l'activité apportés par euxà ces opérations, d'avoir prochainement des données certaines pour baser nos calculs de meilleure utilisation.

En attendant, pour les industriels désireux d'aménager des chutes, il y a déjà un moyen d'évaluer avec une approximation suffisante les débits limites entre lesquels doit osciller le volume d'alimentation des usines projetées.

Lorsqu'il s'agit de rivières soumises au régime alpestre, quelques jaugeages effectués pendant les périodes les plus froides de décembre-janvier $t t$ aux heures du soleil levant. 
donneront, à peu de litres près, le débit minimum minimorum. D'autre part, en appliquant à ces rivières la loi hydrologique approximative: le débit moyen (en lit. par sec.) est égal au produit de la surface (en $\mathrm{km}^{2}$ ) du bassin versant par un coefficient dont la valeur est comprise entre 20 et 30 , on trouve la valeur maximum du débit que l'on peut avoir intérêt à dériver $\left.{ }^{*}\right)$ Je dis la valeur amaximum » car le débit journalier de la rivière sera, pendant les mois d'hiver, en dessous du débit moren annuel ainsi calculé, et en dessus pendant les mois d'été; ce qui est loin d'impliquer cette circonstance que le débit soit pendant six mois supérieur au chiffre fourni par l'application de la précédente loi : il pourra être supérieur à ce chiffre, aussi bien pendant sept à huit mois que durant quatre ou cinq mois seulement; pour chaque rivière cela dépend des saisons et de l'influence des glaciers et des neiges sur le régime hydrologique de son bassin. Suivant l'écart.constaté entre le débit minimum et ce débit moyen, on voit si ce dernier doit être pris comme maximum de la dérivation. Lorsque l'écart est grand, il y a beaucoup de chances pour que le débit moyen ne dure pas six mois et comme (sauf de rares exceptions dans le cas de très hautes chutes) il y a peu d'intérêt à se servir de chevaux périodiques durant moins d'un semestre, il est inutile de prévoir les ouvrages de dérivation pour un cube atteignant la valeur du débit moyen. Quand, au contraire, l'écart n'est pas considérable, on peut en conclure que ce débit sera réalisé pendant plus de la moitié de l'année, et alors il est rationnel, dans le cas des moyennes et hautes chutes, de disposer les travaux de dérivation pour l'utiliser.

Au sujet de l'évaluation des amplitudes du débit de part et d'autre du chiffre qu'il importe de choisir pour maximum de la dérivation, je recommande particulièrement l'étude de M. le commandant Audebrand publiée à cette place, dans le numéro de mai 19o3, sous le titre : "Au 'sujet des débits caractéristiques ». Par l'analyse des causes perturbatrices du débit, on peut, comme il ressort des moyens indiqués dans cette savante étude, connaître le régime d'une rivière sans avoir à faire de très nombreux jaugeages.

L'avantage des moyens proposés par M. Audebrand est, au surplus, qu'ils permettent la prédétermination du régime non seulement des rivières alpestres mais encore de tous les cours d'eau. En particulier, ils peuvent rendre de grands services dans l'aménagement des rivières pyrénéennes où les procédés d'évaluation des débits limites que je viens d'indiquer, quoique applicables dans une certaine mesure, sont beaucoup moins faciles à mettre en cuvre et donneraient des résultats peu significatifs. Enfin, c'est pour les cours d'eau ayant leur éliage en été, où lesdits procédés ne pẹvent plus être employés, que la méthode de notre colla. borateur est surtout recommandable. Elle donne le moyen de déterminer, dans un temps minimum d'observation, le débit qu'il convient de dériver pour obtenir la meilleure utilisation dune chute à débıt variable.

Au reste, l'appréciation des débits limites est facilitée pour

(1) On prend le coefficient 20 (ou voisin de 20) lorsqu'il n'y a pas (ou peu) de glaciers dans le périmètre du bassin de réception; le chiffre 30 est pris, quand, au contraire, le cours d'eau est alimenté par des glaciers. un assez grand nombre de rivières sur lesquelles existent déjà des usines hydrauliques; à l'aide des ouvrages de dérivation de ces usines on a pu se rendre compte du régime des cours d'eau qui les alimentent.

Quant aux rivières où nulle chute n'est encore installée, nous croyons que bien souvent on pourrait, dans le cas de très importantes installations à établir sur leur cours, metıre à exécution ce projet que nous savons formé par des industriels prudents : une fois l'emplacement choisi, procéder à la création de la chute en construisant d'abord seul le barrage de dérivation pour, au moyen de cet ouvrage fonctionnant en déversoir, relever pendant un an les débits journaliers de la rivière. Nous avons nous-mêmes indiqué dans ce but des méthodes et appareils permettant d'obtenir, d'une matière automatique et continue, l'inscription graphique de ces débits. Or, des relevés faits pendant un an, on peut déduire, par application de la théorie de M. AudeBRAND, le régime du cours d'eau à capter.

On peut donc presque toujours si l'on s'en veut bien donner la peine, en attendant la publication des graphiques de débit préparés par la Mission d'étude des grandes Forces hydrauliques, procéder en suffisante connaissance de cause.

Je passe maintenant aux observations suivantes. - Quel que soin que j'aie pris en parlant des hautes, moyennes et basses chutes, de préciser par des exemples numériques la valeur que $j$ 'attribuais à ces dénominations, un certain nombre de lecteurs - et des plus autorisés - m'ont écrit pour me signaler tout l'arbitraire des chiffres limitatifs de catégories indıquées dans mon article. Je ne conteste nullement le bien fondé de leurs critiques mais je me demande comment faire à l'avenir, dans une étude de ce genre, pour désigner tantôt la catégorie des chutes comprises entre o et 20 mètres de hauteur par exemple, tantôt la catégorie comprenant celles de 200 à I ooo mètres de hauteur? Personne ne conteste qu'elles se présentent, au double point de vue économique et technique, dans des conditions d'aménagement et d'utilisation très différentes. Alors pourquoi ne chercherait-on pas à se mettre d'accord sur une classification où chacune des catégories qu'on peut faire serait caractérisée par les conditions économiques de son appropriation et limitée par des chiffres admis de tous; ne seraitil pas plus simple de s'entendre sur ce fait que quand on parle des basses chutes en général, on sait qu'il s'agit de celles dont la hauteur est comprise entre $x$ et $y$ mètres; de même pour les morennes et hautes chutes? Aujourd'hui je constate malheureusement que pour satisfaire tout le monde il faut se donner garde d'employer ces dénominations. Quand on veut désigner des chutes de telle ou telle hauteur, il ne faut pas dire ce sont des hautes ou des basses chutes, mais des chutes de $H$ mètres. Dans ces conditions, le langage de cette branche de l'économie industrielle devient tellement compliqué que c'est à y renoncer.

Plusieurs hydrauliciens ont récemment proposé des clásšifications.

Les uns s'appuyant uniquement sur les conditions de marche des récepteurs hydrauliques n'éprouvent la nécessité que de deux catégories : les basses chutes (de o à 10 ou 12 mètres); les hautes chutes (au-dessus de ro à I 2 mètres). A ceux-là je demande si entre une chute de 800 ou 
900 mètres et une autre de 50 mètres, il n'y a pas, au simple point de vue des moteurs, autant de différence qu'entre cette même chute de 50 mètres et une troisième de 3 ou 4 mètres? Cette classification me paraît insuffisante.

Les autres, en vertu de considérations d'ordre économique proposent, comme M. le commandant Audebrand $\left(^{*}\right)$, des catégories fort nombreuses: très faibles hauteurs (o à I m.); faibles hauteurs ( $\mathrm{m}$. à $10 \mathrm{~m}$.) ; petites hauteurs (10 m. à $100 \mathrm{~m}$.) ; moyennes ( $100 \mathrm{~m}$. à $250 \mathrm{~m}$.) ; grandes $(250 \mathrm{~m}$. à 500 m.) ; (rès grandes (500 à $750 \mathrm{~m}$.) ; hauteurs exceptionnelles (au delà de $750 \mathrm{~m}$.). Si rationnelle et ingénieuse que soit la justification de cette échelle, je trouve ses échelons un peu trop nombreux pour apporter une simplification dans le langage courant.

Il vaudrait mieux, à mon avis, prendre un moyen tcrme entre ces extrêmes: in medio stat pirtus l... Par division de l'échelle des chutes en ces trois catégories: basses, moyennes et hautes, il me semble qu'on pourrait clairement les caractériser toutes au double point de vue économique et technique. Mais je laisse à d'autres le soin de nous mettre d'accord sur les chiffres limitatifs de ces catégories à fixer.

Impossibilité d'employer les chevaux périodiques seuls à 1'électrochimie? - Les plus sérieuses objections qui m'ont été faites sont relatives à cette idée, à tort déduite de mon exposé, que je considère l'électrochimie comme devant être alimentée seulement par les chevaux périodiques; et elles peuvent toutes se résumer en ceci : "Vous calculez les chevaux permanents à l'aide du débit caractéristique d'éliage, tel que l'a défini M. R. Tavernier; or, c'est par définition "le débit au-dessous duquel le cours d'eau descend pendant dix jours par an au plus ». Donc, pendant dix jours au moins par an, il n'y aura pas de chevaux périodiques et l'électrochimie sera sûrement arrêtée ; et en outre, de part et d'autre de cette période de dix jours, il existera des semaines, surtout dans les années sèches, où ces chevaux seront si peu nombreux qu'ils ne suffiront pas à assurer la marche, même très ralentie, de l'usine électrochimique ; bien mieux, pendant dix jours par an, il n'y aura même pas assez de chevaux permanents pour passer les pointes d'extrême charge d'un réseau de transport d'énergie si vous comptez que la puissance fournie par le débit caractéristique d'étiage, est le reste du' temps, suffisante pour alimenter ce réseau. Les industries électrochimiques peuvent, en général, s'accommoder de quantités très variables d'énergie, mais assez rares sont celles qui supportent un chômage complet durant plusieurs semaines consécutives. Par conséquent, on ne peut pas faire à la fois du transport de force et de l'électrochimie dans les conditions que vous dites; votre théorie est inapplicable. )

Je n'hésite pas cette fois à reconnaître que l'objection porte et nécessite pour être levée des explications précises. Toute l'ambiguité de mon argumentation provient de l'emploi mal à propos du débit caractéristique d'étiage. Je ne veux en aucune manière critiquer ce paramètre introduit par M. Tavernier dans l'étude hydrologique des cours d'eau; il est très ingénieux et d'une énonciation fort com-

(*) Voir La Houille Blanche, no d'août rgo2. "Réflexions sur les études préliminaires à l'aménagement des chutes d'eau $₫$. mode pour caractériser des périodes du régime d'une rivière, celle qui est de beaucoup la plus importante à considérer au point de vue de son utilisation; mais il ne faut pas en user comme d'une règle à calcul servant à faire quantité d'opérations. Je l'avais modifié pour les besoins de la cause en disant que le débit caractéristique d'étiage est celui au-dessous duquel le cours d'eau descend pendant dix jours en l'année la plus sèche de dix années consécutives: cette modification a peu changé à mon raisonnement et je précise ce que j'ai voulu dire.

Admettons que nous ayions à faire l'aménagement d'une chute sur une section de rivière où un devis d'avant-projet nous a conduit à fixer à 100 mètres la dénivellation la plus favorable. Les jaugeages faits journellement du 15 novem. bre au I 5 mars, l'année s'étant trouvée - circonstance fort heureuse - sèche et très froide, nous ont donné le gra* phique de débit représenté fig. I. Ce graphique nous

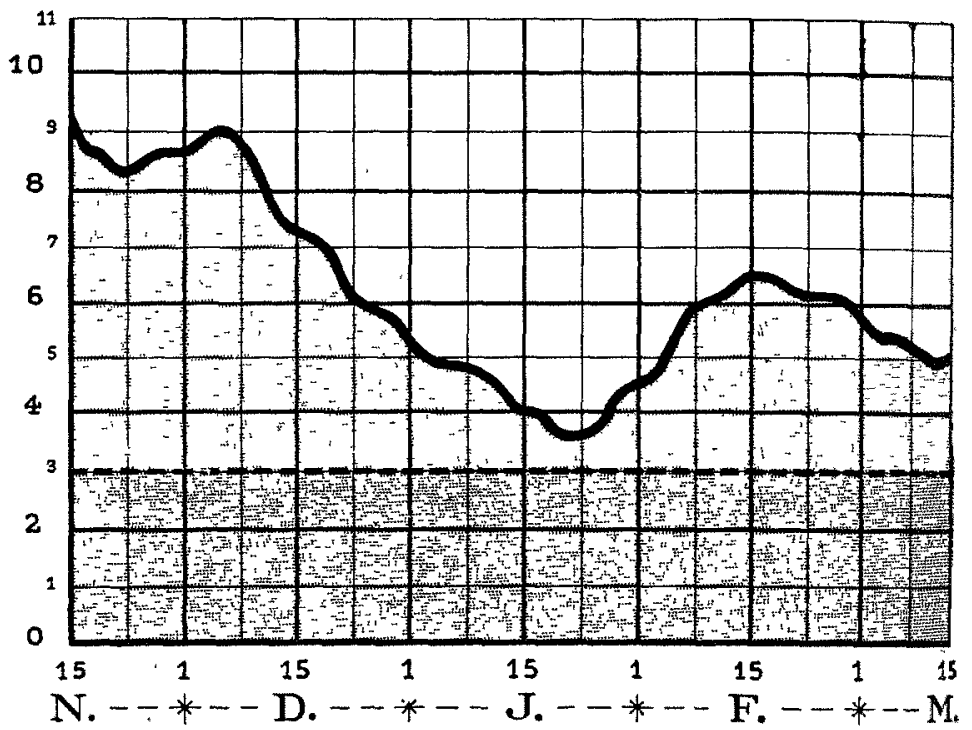

Fig. I. - Diagramme des débits d'une rivière à régime alpestras dans la pérjode où se produit le débit minimum.

apprend que le minimum s'est produit dans la deuxième quinzaine de janvier. Probablement, les autres années, il a lieu un peu plus tôt ou un peu plus tard, mais la rivière étant sous la dépendance de glaciers, le minimum obserté en cette année sèche et en cette saison exceptionmellement froide paut être considéré à peu de litres près, comme le minimum minimorum. Il durera plus ou moins longtemps, mais ne persisterait-il que quelques heures nous ne calciv lerons pas nos chevaux permanents d'après un débit supé rieur à ce minimum. Et même pour plus de prudence alors que nous le trouvons égal à 3500 litres, nous ne base* rons quand même le calcul des chevaux permanents que surt un débit légèrement inférieur, soit sur 3 ooo litres. Cette précaution est justifiée, non seulement par l'incertitudeda minimum minimorum, mais encore par le défaut d'approximation des jaugeages : même en supposant qu'on fasse ces opérations sur un simple déversoir, on peut très bien contmettre une erreur de 10 pour I oo et n'avoir que 3000 et quelques litres au lieu de 3500 . Il est vrai qu'on peut faire l'erreur inverse. Dans le doute nous prenons le minimum. Pendant toute l'année nous aurons donc:

$$
\frac{3000 \times 100}{7^{5}} \times \frac{75}{100}=3000 \mathrm{HP} \text { permanents, }
$$


et nous sommes certains de les avoir d'une façan ininterrompue tous les ans.

En procédant ainsi, nous pouvons en toute sécurité affecter ces chevaux à l'alimentation d'un service public. Soit (fig. 2) les diagrammes de charge journalière que d'après des renseignements précis l'on peut prévoir au tableau de distribution de l'usine à construire pour alimenter ce service public. Voici comment je suppose que ces diagrammes ont été obtenus. Dans une ville $\mathrm{N}$ existe $: 1^{\circ}$ une ligne de tramways produisant elle-même son énergie par une usine à vapeur - on possède ses diagrammes de charges journaliers $-; 2^{\circ}$ une station à vapeur d'éclairage et de force motrice électriques - on possède également ses diagranmes de consommation.- Ces deux compagnies se sont mis d'accord pour substituer intégralement la houille blanche à la houille noire et sous cette condition absolue que leur com. mun fournisseur d'énergie hydro-électrique leur assurera en tout temps le nombre de kilowatts nécessaire pour n'avoir recours à leurs appareils à vapeur que dans le cas d'un accident à l'usine génératrice ou sur les lignes de transport d'énergie.

Nous avons donc pris, dans chacune de ces compagnies, les diagrammes de charges maxima, et nous avons additionné la consommation la plus élevée des jours d'hiver à l'usine des tramways à la consommation journalière également la plus élevée de l'usine d'éclairage et de force; puis nous avons répété la même opération pour les mois de juin, juillet, août; en ajoutant à ces sommes les pertes des lignes qui doivent réunir l'usine hydro-électrique à chacune des précédentes stations, nous avons obtenu les dia. grammes de la figure 2 . Le trait continu représente, au tableau de l'usine hydro-électrique, les charges maxima d'hiver, et le trait pointillé les charges maxima des 30 jours les plus longs de l'année (*). Leur interprétation se conçoit aisément. Entre minuit et une heure du matin les tramways s'arrêtent et l'éclairage privé s'éteint; la courbe de consommation baisse. De une heure à 4 heures du matin, été comme hiver, seul l'éclairage public fonctionne et la courbe passe par son minimum. De 4 à 6 heures les premières voitures de tramways entrent en circulation, mais un peu plus tôt en été qu'en hiver; par contre en hiver, un grand nombre de lampes sont allumées dans les boutiques, atcliers et magasins qui ouvrent de bonne heure, de sorte que les deux courbes montent ensemble jusqu'à 6 heures, mais entre 6 et 9 heures la courbe d'hiver passe par un maximum assez élevé. De $g$ heures à midi, il n'y a pas d'éclairage, mais tramways et moteurs chargent simultanément le réseau à peu près de la même quantité l'hiver et l'été. Entre midi et 2 heures une partie des ateliers ferment, les moteurs s'arrêtent et les deux courbes subissent une dépression marquée. A partir de 4 heures en hiver l'éclairage public et privé, surtout celui des magasins et bureaux, intervient pour charger au maximum le réseau et provoque la pointe très accusée s'élevant beaucoup au-dessus du diagramme d'été, lequel représente seulement pen-

( $)$ Ce dernier diagramme a peu d'importance dans le cas qui nous occupe, mais nous l'avons établi parce que noús aurons à en reparler un peu plus loin. dant ces heures la consommation des tramways et des moteurs. A 8 heures, les ateliers, les bureaux et un certain nombre de magasins sont fermés et les courbes de charge descendent, mais beaucoup plus brusquement en hiver qu'en été. De 10 heures à minuit, si les voitures de tramways sont moins nombreuses en circulation, par contre l'éclairage des cafés et des salles de spectacle relève les diagrammes.

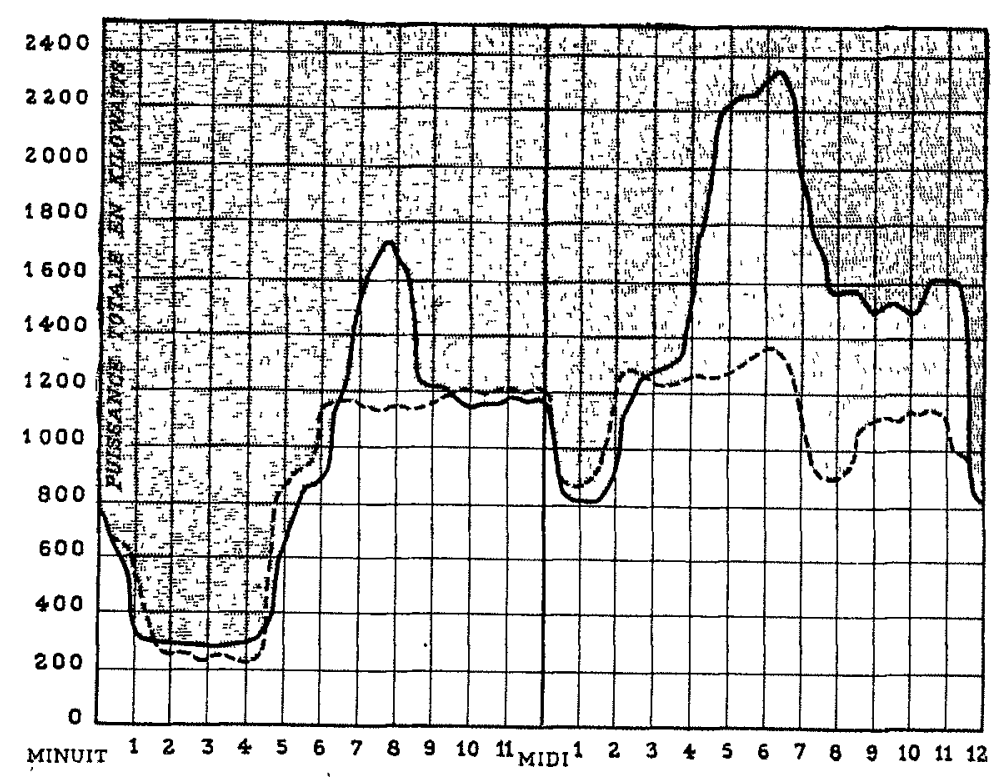

Fig. 2. - Diagrammes des charges journalières d'une distribution d'énergie à alimenter par les chevaux permanents d'une usine hydro-électrique.

Trait plein : Courbe de charge maximum d'une journée d'huver.

Trait pointillé : Courbe de charge d'une journce d'été.

Comme un le voit la consommation maximum du réseau s'élève à $2300 \mathrm{kw}$ environ. Elle n'atteint pas tous les jours d'hiver cette puissance, mais pendant un bon mois elle s'éloigne très peu du maximum constaté. Celui-ci dure fort peu de temps, une demi-heure au plus, mais aurait-il une durée plus courte encore, il faut que les chevaux permanents puissent, en toute circonstance, passer la pointe formée par ce maximum. Autrement dit, si l'on considere simultanément les figures I et 2 , il faut que le diagramme de charge maximum en hiver s'inscrive tout entier dans l'aire (la plus teintée de la fig. 1) limitée par l'axe des abcisses et la parallèle pointillée passant par l'ordonnée qui donne 3 ooo litres et au-dessus de laquelle se tient toujours le débit du cours d'eau.

Dans notre exemple, cette condition n'est même pas complètement remplie puisque la puissance sur l'arbre des turbines est de 3 ooo HP, la charge maximum du réseau $2300 \mathrm{kw}=3125 \mathrm{HP}$ et qu'il faut encore tenir compte du facteur de puissance. Ceci nous amène à prendre quelquefois les 3500 litres accusés par le minimum du graphique de débit. Et comme ce minimum a lieu précisément dans les jours d'hiver où la pointe du diagramme de charge passe par.son maximum, il arrive parfois que les deux courbes sont tangentes. Or, c'est commettre une imprudence que de réaliser cette tangence, et je n'ai choisi cet exemple que pour mieux appuyer ma maniere de voir.

De ceci je conclus que le débit caractéristique qu'il importe le plus de connaître; ce n'est pas celui au-dessous 
duquel le cours d'eau descend pendant dix jours par an; année quelconque, mais bien le débit minimum-minimorum. C'est, à mon avis, le seul sur lequel on puisse sérieusement baser un calcul d'alimentation de service public. (Quant au débit pouvant servir à caractériser la limite d'utilisation de la puissance variable du cours d'eau, nous verrons tout à l'heure ce qu'il peut être suivant les cas). Ce débit minimum-minimorum est assez difficile à déterminer d'une manière certaine. Je demande à ceux qui s'occupent d'hydrologie si l'on peut le déduire aveć assez de certitude de l'examen des graphiques journaliers de débit d'un cours d'eau relevés pendant dix années consécutives? Dans l'afirmative ce serait là un paramètre invariable qu'on pourrait introduire en toute sûreté dans les calculs. Pourquoi alors n'appellerait-on pas simplement ce débit caractéristiqui: le débit minimum?

Par le rapprochement que nous venons de faire des deux diagrammes précédents on voit que :

$1^{\circ}$ Une bonne partie des chevaux permanents n'est pas utilisée. Toute la surface comprise entre la parallèle à l'axe des abcisses passant par l'ordonnée $2300 \mathrm{kw}$ et le contour du diagramme de charge maximum (partie la plus teintée de la fig. 2), c'est-à-dire près de la moitié de la puissance de l'usine génératrice, est inutilisée en hiver. A cette surface s'ajoutent encore en été les aires les moins teintées comprises entre les deux courbes, pleine et pointillée. Si l'on faisait la sommation de tous les diagrammes journaliers on trouverait que le nombre moyen de kilowatts-24 heures consommés est inférieur à la moitié du nombre de $\mathrm{kw}-24 \mathrm{~h}$. en puissance à l'usine. Ces chevaux permanents non utilisés, au moins 22 heures sur 24 et pendant 8 à 10 mois de l'année, constituent ce, qu'on a communément appelé les résidus d'énergie. Mais, selon moi, c'est là seulement la fraction d'énergie non utilisée qu'il convienne de désigner sous ce nom. Il ne faut pas considérer comme "résidu d'énergie " la partie variable de la puissance dont nous allons maintenant parler. Nous

verrons plus loin comment on peut réduire à son minimum la part de cette énergie résiduelle.

$2^{\circ}$ Les chevaux périodiques représentés par la surface la moins teintée de la fig. I, comprise entre la parallèle pointillée correspondant à $3 \mathrm{~m}^{3}$ et la courbe des débits journaliers, sont dans le cas qui nous occupe, alors même que nous considérons seulement la partie basse du graphique annuel, en nombre au moins égal à celui des chevaux permanents : la sommation des deux parties de ce diagramme donnerait un nombre moyen de HP périodiques-24 heures un peu plus grand que le nombre journalier de HP permanents. Si au lieu de restreindre cette comparaison à la période annuelle des quatre mois de plus faible débit ( 15 novembre au I 5 mars, fig. I) on l'étend à l'année entière, on voit (fig. 3 - le gros trait représentant alors le diagramme des débits durant toute l'année $\rightarrow$ ) que les che. vaux périodiques forment la partie de la puissance de beau. coup la plus massive dont il s'agit de savoir que faire.

Ceci nous conduit à entrer maintenant dans le vif de la question qui est l'objet principal de cette étude.

L'exemple choisi de graphiques de débit, représentés fig. 3, est ce que je demande la permission d'appeler, pour la généralisation du raisonnement, un exemple moren. Je veux dire par là qu'au lieu de se rapporter à une seule rivière, ils ont été construits en prenant les moyennes de débits qui ont été relevés les mêmes jours sur plusieurs rivières soumises au régime alpestre. Nous pouvons donc admettre que les courbes de cette figure 3 donnent une idée suffisamment exacte de l'allure du débit en l'une quelconque de nos rivières alpestres et que ce qui est vrai dans le cas de cet exemple moyen reste vrai dans le cas particulier de chacune de ces rivières.

Cela étant donc admis, revenons à l'argumentation du

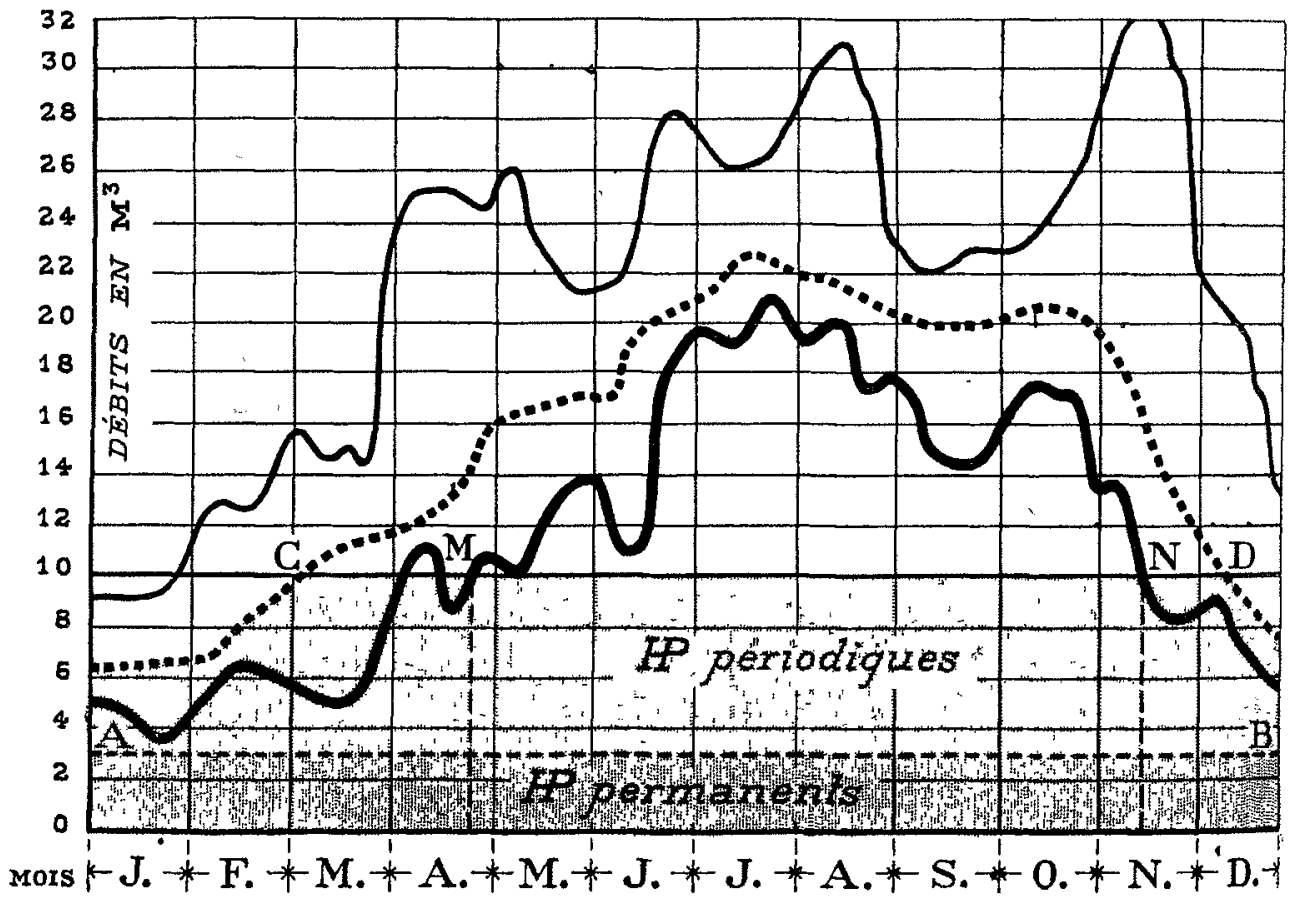

Fig. 3. - Diagrammes des débits annuels d'une rivière type soumise au régime alpestre.

Gros trait: Courbe des débits pendant la plus sèche de dix années consécutives.

Trait fin: Courbe des débits pendant une année de crues.

Trait pointillé : Courbe de la moyenne des débits pendant dix années consécutives. paragraphe I de cet article; l'examen de la fig. 3 la rend plus claire. Dans le cas des basses chutes le cube à dériver en sus du débit minimum est tel qu'il rend le cheval périodique très cher et étant donné l'irrégularité de travail de ce cheval on n'en peut trouver l'emploi qu'exceptionnellement; on n'aménagera donc que les chevaux permanents. Dans le cas des hautes chutes le cube à dériver en sus du débit minimum étant faible, rend le cheval périodique très bon marché et qu'on en ait ou non l'emploi immédiat il faut l'aménager. Entre ces deux cas extrêmes il existe toujours; on le conçoit, une solution répondant au principe de la meilleure utilisation. Or, par l'exemple indiqué plus haut, chute de soo mètres de hauteur, nous nous sommes précisément placés entre ces extrêmes. Pour quel débit maximunt 
avons-nous intérêt à établir nos ouvrages de dérivation? J'ai répondu: pour un débit tel qu'il donne, en sus des chevaux permanents, une puissance invariable pendant al moins 180 à 200 jours consécutifs en année sèche; de la sorte nous pourrons toujours, étant donné le bas prix de revient de cette énergie, l'employer un jour ou l'autre à des opérations électrochimiques ou électrométallurgiques. Les diagrammes, fig. 3 (le gros trait représentant la courbe des débits de l'année la plus sèche de Io années consécutives), nous montrent que ce débit doit être de $\mathrm{Io}^{\mathrm{m}}{ }^{3}$. Les points $\mathrm{M}$ et $\mathrm{N}$ à l'intersection de la courbe et de la droite dont l'ordonnée représente un débit de $10^{\mathrm{m} 3}$ sont distants de 200 jours au moins pendant lesquels le débit ne tombe pas au-dessous de cette valeur.

Voici donc quel est le débit maximum que dans le cas de l'exemple choisi nous avons intérêt à dériver. On voit de suite que non seulement il ne sera pas le même pour chaque cours d'eau, mais encore que sur une même rivière il variera pour chaque chute selon la dénivellation mise en ouvre. Dans ces conditions est-il bien nécessaire de chercher à définir un débit caractéristique d'utilisation maximum? Tout de même, au point de vue du recensement de nos forces hydrauliques. Mais on verra par ce qui suit qu'il conviendrait de le fixer à une valeur inférieure au débit moyen et de préférence égale à celui au-dessus duquel le cours d'eau reste pendant huit mois au moins en année moyenne. Par simplification on pourrait l'appeler: dobit industriel.

C'est sur l'affectation à l'électrochimie de ces chevaux périodiques ici contenus dans l'aire délimitée, à la partie inférieure par la ligne pointillée $\mathrm{AB}$, à la partie supérieure par la parallèle MN et latéralement par les deux sections inférieures de la courbe des débits en année sèche, qu'on a attaqué mon argumentation.

Certaines industries et notamment l'électrométallurgie, m'objecte-t-on, exigent pour soutenir victorieusement la lutte qu'elles ont engagée contre les vieux procédés, la fourniture de grandes quantités d'énergie et surtout l'emploi d'une puissance constante. Leurs opérations doivent être conduites par un personnel spécialement instruit, aux appointements élevés, qu'on ne peut, sans de graves préjudices à tous égards, congédier à certaines époques de lannée et qu'il fauten conséquence maintenir constamment en cuvre. Le capital d'une installation électrochimique ou électrométallurgique est toujours considérable ; en raison de la rapide usure des appareils d'utilisation du courant électrique et surtout à cause des incessantes modifications qu'y nécessite: le progrès, ce capital appelle un amortissement à court terme; on ne saurait donc laisser ces installations inactives durant de longues périodes. Enfin le fonctionnement de ces appareils ne peut être interrompu qu’à bon escient, au moment voulu, et il ne doit jamais souffrir d'une irrégularité dans la fourniture du courant; les appareils d'utilisation commandent les génératrices d'énergie et ne s'accommodent point d'intempestives variatıons de puissance. Telles sont résumées en quelques lignes les raisons données pour combattre mon raisonnement.

Considérées en elles-mêmes ces raisons sont très justes et je ne les ignorais pas. Mais j'estime qu'il faut envisager à un autre point de vue, l'économie industrielle de l'électrochimie. Qu'est-ce qui importe le plus à une usine électrochimique ou électrométallurgique? C'est sans conteste d'avoir le cheval au plus bas prix possible; là est la condition sine qua non de son existence et de sa prospérité. L'économie réalisée dans les prix de revient de fabrication par le bas prix de l'énergie est en général plus grande que celle qui résulte d'un amortissement du capital uniformément réparti sur une production régulière mais obtenue au moyen d'une énergie coûteuse. C'est ensuite d'avoir tou- 
pendant 240 jours égal ou supérieur à ${ }_{1} 2^{3}{ }^{3}$; pendant 120 jours égal ou supérieur à $20^{\mathrm{m}^{3}}$, etc... On voit en particulier que pendant 276 jours (intervalle de temps compris entre les points $C$ et $D$ de la courbe pointillée de la figure 3) le débit industriel est de $10^{\mathrm{m}^{3}}$. Pendant tout ce temps, en année moyenne, nous disposerons donc de: $\frac{\left(10000^{1}-3000^{1}\right) \mathrm{I}^{\mathrm{m}}}{7^{5}} \times \frac{7^{5}}{100}=7000 \mathrm{HP}$ périodiques. De plus, pendant $360-276$, soit 84 jours, nous , pourrons disposer en moyenne d'un débit de 7000 - 3000 litres, c'est-à-dire 4000 litres en sus du débit minimum, ce qui nous donnera encore $4000 \mathrm{HP}$ périodiques.

Pendant l'annnée nous aurons done:

$360 \times 3000=1080000 \mathrm{HP}$ permanents -24 heures; et $276 \times 7000+84 \times 4000=2268000 \mathrm{HP}$ périodiques -24 heures.

Pour couvrir ses frais d'exploitation (comptes: personnel, intérêts, amortissements et entretien réunis) et s'assurer un bénétice annuel de $n \%$, l'usine génératrice du courant électrique est obligée de compter sur une recette brute de 468 ooo francs. Si elle a été appareillée uniquement en vue de fournir à une installation électrochimique la totalité de ses chevaux permanents et de ses chevaux périodiques elle ne peut, dans ces conditions, opérer ladite fourniture qu'à raison de o fr. 44 le cheval-24 heures (suit 50 fr. le FIP-an). Mais si, au contraire, elle peut vendre à un transport d'énergie ses 3 ooo HP permanents à raison de $\mathrm{I}$ oo $\mathrm{fr}$. le HP-an au tableau de distribution, elle se fait de ce chef un revenu brut de 300 ooo francs. Il ne lui reste donc plus, pour atteindre son but financier, qu'à récupérer 468 o00 $300000=168000$ francs sur les $2268000 \mathrm{HP}$ périodiques-24 heures livrés à l'installation électrochimique. Cela met alors alors le cheval périodique-24 heures à 7 cent. 4 , soit moins de 27 francs le cheval-an. Et de ce fait l'installation électrochimique économise :

$$
2268000 \text { (of } 140-\text { of } 074 \text { ) }=\text { I } 49688 \text { francs. }
$$

Cette économie sur la consommation d'énergie n'est-elle pas de nature à compenser dans l'exploitation électrochimique certains inconvénients qui peuvent résulter d'une marche non absolument régulière ? - Je me demande même pourquoi je me suis laissé aller à produire ce calcul de petit écolier: le fait indiqué apparaît de lui-même avec la plus grande évidence $\left({ }^{*}\right)$.

Quant àl'irrégularité du travail des chevaux périodiques, je ne crois pas qu'on puisse la considérer comme un obstacle au seul emploi de ceux-ci en électrochimie. Les chevaux permanents ayant été calculés comme il a été dit plus haut, les graphiques de débit nous montrent que, même dans les années d'extrême sécheresse, ils ne nous feront jamais complètement défaut pendant un mois entier; qu'en année

(*) Cela est si vrai que certaines usines où, seuls ont été aménagés les chevaux permanents, mais à un prix relativement bas grâce à dès conditions de captage exceptionnellement favorables, font a la fois du transport de force et de l'électrochimie, et cela pour abaisser au moyen du revenu des cheraux-24 heures livrés aux établissements électrochiniques, le prix de l'énergie distribuée aux services publics pour éclairage et force motrice. Un exemple typique de cette solution est fourni par les usines de Rheinfelden, sur le Rhin; dont il a été párlé dans le précédent numéro de la Revue. moyenne ils seront toujours en nombre à peu près égal à celui des 'chevaux permanents, et le plus souvent ne varieront que du simple au double. Ce n'est peut-être qu'une année ou deux sur dix qu'ils manqueront totalement durant quelques semaines consécutives où à interralles très rapprochés. Mais encore cette période d'arrêt pourra-t-elle être prévue. Les variations de débit d'un cours d'eau obéissent à des lois générales que peuvent faire connaître et l'étude hydrologique suffisamment prolongée de son bassin versant et les observations météorologiques organisées dans ce but. Quoique encore peu nombreux aujourd'hui, les postes d'observation destinés à fournir aux riverains ces prévi. sions hydrologiques vont se multiplier et je ne doute pas qu'à l'aide de moyens assez faciles à concevoir, ils ne parviennent à prévoir à plusieurs semaines d'échéance les variations importantes du ftux des rivières. On ne peutdonc pas dire qu'en adoptant la solution préconisée du problème de la meilleure utilisation, les usines électrochimiques seront exposées à des arrêts longs ou intempestifs, puisqu'ils ne peuvent se produire qu'à une époque déterminée, à peu près fixe dans l'année, et dont on parviendra d'avance à déterminer la date.

Ces arrêts, nous allons, dans la seconde partie de cet article, indiquer les moyens de les éviter d'une manière complète dans bon nombre de cas, mais en admettant qu'on ne puisse y réussir s'en suit-il, abstraction faite des considéra. tions précédentes, que les industries électrochimiques" ne s'en accommoderont jamais ? - Voyons un peu s'il n'y a pas de grandes industries, ayant au point de vue économique quelque analogie avec celles de l'électrochimie et de l'électro. métallurgie, qui chôment périodiquement tous les ans.

Les sucreries, comme on le sait, travaillent seulement de novembre à fin février, leur "campagne " ne dure que quatre mois. - Les verreries abattent les feux de leurs fours pendant les mois de juillet et août. - Les fabriques de chaux hydrauliques et ciments ont des périodes de marche considérablement ralentie chaque année en décembre-janvier et juillet-août. - Et combien d'autres exemples ne pourraiton pas citer encore?

Toutes ces usines n'ont-elles pas un personnel de techniciens qu'elles ne congédient jamais, des frais d'amortissement énormes, etc...? Profitant des jours de chômage pour réparer leurs appareils ou leurs fours, elles occupent à ces travaux d'entretien leur personnel fixe; seuls, les ouvriersmancuvres sont embauchés et débauchés à chaque campagne; elles franchissent ainsi sans inconvénients ces périodes d'arrêts ou de ralentissement; et même n'existeraient-elles pas du fait d'influences extérieures que ces usines y seraient amenées par l'obligation de remettre en état leur matériel, à moins de l'avoir en double, ce qui nécessiterait parfois un capital très lourd à rémunérer et amortir. - Ne peut-il pas en être de même dans les futures usines électrochimiques et électrométallurgiques ? Sans doute, répondra-t-on, mais les sucreries, les verreries et fabriques de ciment ont toutes leur marche assujettie aux mêmes circonstances commerciales ou techniques motivant ces chômages; dans la concurrence qu'elles se livrent leurs moyens de lutte sont égaux. Il n'en est plus de. même en électrochimie. L'usine qui utilisera seulement les 
chevaux périodiques sera en état d'infériorité vis-à-vis des usines déjà installées, employant aux mêmes opérations toute l'énergie de chutes puissantes et peu variables.

Evidemment, il y aura toujours quelques usines plus privilégiées lés unes que les autres et il ne saurait en être dans ces nouvelles branches de l'industrie, autrement que partout ailleurs. Mais ces usines privilégiées sont-elles si nombreuses? On peut même se demander si elles ne s'empresseraient pas d'offrir leurs chevaux permanents à un transport d'énergie, au cas où celui-ci deviendrait possible par suite de la création de nouveaux réseaux, et si l'impossibilité où elles pourraient se trouver de le faire ne constitue pas l'inverse d'un privilège ( $\left.{ }^{*}\right)^{t_{*}}$. Dans cette étude je ne puis pas ne considérer que l'état actuel de ce débouché de la houille blanche. Depuis quinze ans, 100000 chevaux ont été installés pour faire de l'électrochimie, mais il n'y en a réellement que la moitié environ d'affectés à l'usage prévu; l'autre moitié change peu à peu de destination et passe au transport de force toutes les fois que l'occasion s'en présente. Or qu'estce que ces 50 à 60 ooo chevaux d'usines électrochimiques devant les i 500000 à 2 millions qui restent à employer par l'électrochimie et l'électrométallurgie?

Souhaitons que le progrès de ces industries avance parallèlement à celui du transport d'énergie à grande distance. Car ce qui s'opposera le plus à la solution du problème de la meilleure utilisation sera suivant que l'un ou l'autre de ces progrès, ira le moins vite : ou bien l'impossibilité pour une usine hydro-électrique qui aura le placement de ses chevaux permanents sur un réseau de distribution, de trouver l'emploi de ses chevaux périodiques, faute d'applications électrochimiques; ou bien, inversement, l'impossibilité pour une usine d'électrochimie ou métallurgie de trouver l'emploi rémunérateur de ses chevaux permanents, faute de pouvoir se connecter avec un réseau de transport d'énergie. Cette solution n'est aujourd'hui réalisée qu'à l'état d'exception parce que les circonstances économiques desquelles elle dépend ne sont point encore nées; mais le jour où le transport de force et l'électrochimie auront donné tout ce qu'on est fondé à attendre d'eux, elle sera la règle.

(7) Je sais qu'une usine électrométallurgique, celle entre autres qui aura des hauts-fourneaux opérant la réduction des minerais de fer par exemple, devra disposer en tout temps d'une puissance cónsidérable, chacun de ces appareils absorbant des milliers de chevaux et ne pouvant, sous peine de destruction, être arrêté inopinément. Mais d'abord ces usines constituent actuellement une exception et, ensuite, de l'avis des métallurgistes les plus autorisés, le haut-fourneau électrique de lavenir sera celui qui répondra à ce desideratum: " Augmentation de la rapidité de fusion et partant de la production, "dans le but d'arriver économiquement à une marche discontinue "qui, de ce fait, deviendrait élastique et éviterait la formation des " loups entrainant la perte des appareils actuels (hauts-fourneaux à " houlle noire); de plus, dans ces conditions, le travail de nuit serait "probablement supprimé n. Brouzét. (Les desiderata à réaliser dans la réduction des minerais de fer au four électrique). La Houille Blanche, juin 1902 .

- Il-ne faut donc pas uniquement se baser sur ce qui peut exister àl'heure actuelle, mais bien:sur ce que la marche rapide du progrès nous autorise à concevoir

\section{EMPLOI DES RÉSIDUS D'ÉNERGIE}

Moyens de passer les pointes. - J'ai dit ce qu'il fallait, en considérant les diagrammes de charge d'un réseau de distribution, regarder comme un résidu d'énergie. Il est dû à la pointe d'extrême charge qui a lieu en hiver pendant quelques heures et dont le maximum se produit généralement entre 5 et 7 heures du soir. Sans cette pointe et si par exemple on n'avait jamais que la courbe de charge des journées d'été, toutes les parties teintées de la fig. 2 représenteraient une énergie disponible; les chevaux permanents comme nous les avons calculés n'auraient à fournir au service public que I 400 kilowatts (cas de l'exemple précité) et il nous resterait : $3000 \mathrm{HP}-\frac{1400 \mathrm{~kW}}{0,736}$ $=\mathrm{I}$ ooo HP environ. Ceux-ci pourraient alors être passés au service électrochimique de l'usine qui serait ainsi assuré d'avoir toujours I ooo chevaux permanents, de n'être par conséquent pas arrêté au moment des basses eaux pendant les années d'exceptionnelle sécheresse. La pointe empêche évidemment d'affecter à l'électrochimie cette puis. sance non disponible deux ou trois heures durant, tous les jours d'hiver, ce qui au contraire pourrait se faire dans le cas où on la passerait au moyen d'un artifice approprié.

Je n'ai donc pas besoin d'insister sur l'importance qui s'attache à la réalisation de cette combinaison, mais je crois pourtant bon de faire la remarque suivante: Supposons que ces i ooo chevaux, d'un secours si précieux dans le cas qui nous occupe, soient vendus à l'exploitation électrochimique $25 \mathrm{tr}$. seulement le cheval-an (au lieu de $27 \mathrm{fr}$. que nous avons admis plus haut); cela nous donne une recette annuelle de $25000 \mathrm{fr}$. Elle vaut la peine qu'on s'en occupe, c'est-à-dire que l'on crée pour passer la pointe une installation ou un matériel ad hoc. Or, jusqu'à quelle somme peut s'élever le prix de l'installation à faire dans ce but? - Différents systèmes s'offrent à l'esprit ; suivant que leur coût d'établissement sera en dessous ou en dessus de la somme en question, on pourra les employer ou les rejeter. Or, cette recette annuelle de $25000 \mathrm{fr}$. regardons-la comme devant faire face à deux dépenses, savoir: $I^{\circ}$ l'annuité d'amortissement - très rapide - de l'installation, et cela en raison des appareils d'entretien délicat qu'elle peut comporter, mettons donc un amortissement en 10 ans, soit une annuité égale à $10 \%$ du capital; $2^{\circ}$ l'intérêt à $5 \%$ de ce capital pendant la durée de l'amortissement. Comme ce calcul est évidemment élastique je ne fais pas entrer en ligne de compte la formule des annuités et intérêts composés. La somme limite $x$ que nous cherchons peut se tirer simplement de l'égalité :

$$
\frac{(\mathrm{ro}+5) x}{\mathrm{I} 00}=25000 \quad \text { d'où } x=167000 \text { francs. }
$$

Si l'on venait dire sans préambule à un industriel qu'il doit augmenter de 150000 francs le matériel de son installation pour passer une pointe de 1000 clievaux pendant 2 heures, peut-être trouverait-il la proposition paradoxale! Le raisonnement indique cependant que, dans les conditions où nous le supposons placé, son intérêt est de faire cette augmentation. Elle lui permet d'abord de réaliser en toute sécurité le problème de la meilleure utilisation et ensuite d'avoir, au bout de quelques années, une recette 
supplémentaire non négligeable : 25 ooo fr., moins les frais relatifs au fonctionnement normal du système adopté. Il s'agit maintenant de choisir ce système tel que lesdits frais de fonctionnement soient aussi faibles que possible par rapport à la recette due à son intervention.

Parmi les moyens de passer la pointe qui se présentent à l'esprit, celui consistant à employer une machine à vapeur ne doit pas retenir notre attention. Outre son prix d'installation bien voisin du chiffre limite - précédemment trouvé - un groupe électrogène à vapeur de I 000 chevaux ne travaillant que deux ou trois heures par jour entraîne des dépenses de combustible et de personnel hors de proportion avec le résultat à atteindre. Il faudrait entretenir le feu des chaudières pendant 20 à 22 heures pour quelques heures seulement de marche par jour, et en admettant même que la dépense de charbon (entretien du feu et remise en pression compris) ne dépassât pas $\mathrm{I} \mathrm{kg}$. de charbon par HP-heure effectif, on brûlerait au moins 2000 kilos de charbon par jour, soit dans les quatre mois d'hiver où la pointe se produit, 200 à 250 tonnes de charbon coûtant au bas mot 5000 francs. En ajoutant à cela le graissage et la paye des chauffeurs pendant les jours de marche on arriverait à une dépense d'environ $7000 \mathrm{fr}$.

La seule raison d'être d'une telle manière de passer' la pointe pourrait se trouver dans l'emploi de cette machine à vapeur à d'autres usages. Mais on entrevoit difficilement ces usages: pendant 7 ou 8 mois de l'année l'absence de pointe laisse libre les $\mathrm{I}$ ooo chevaux de la partie supérieure du diagramme de charge, et il suffit pour la rendre disponible l'hiver de quelques kilowatts-heures par jour; que faire du groupe à vapeur quand il les aura fournis, l'eau étant en suffisante abondance le reste du temps?

La question est toute différente si l'on envisage la réguilarisation proprement dite d'une chute par des machines à vapeur. Cette chute étant affectée tout entière (chevaux permanents et périodiques) à un transport d'énergie, la houille noire dans ce cas servira non seulement à passer les pointes mais encore et surtout à remplacer les chevaux périodiques manquant aux basses eaux. Or là n'est pas le problème dont nous poursuivons en ce moment la solution et si intéressant qu'il soit nous le réservons pour. un nouvel article, afin de ne pas interrompre l'examen des autres manières d'utiliser les résidus d'énergie.

Un second moyen peut se trouver dans l'emploi des accumulateurs électriques. Pendant les périodes de faible charge une commutatrice, si le transport d'énergie a lieu par courants alternatifs, ce qui est le cas général, transformera la quantité voulue du courant des alternateurs en marche pour charger une batterie d'accumulateurs; au moment de passer la pointe cette batterie restituera sa charge au réseau par l'intermédiaire de la commutatrice fonctionnant en sens inverse. Ces appareils peuvent être installés soit à la station génératrice, soit à l'extrémité de la ligne de transport, dans une sous-station de distribution. Le principe d'un tel procédé est bien connu et point n'est besoin non plus d'insister sur les détails de sa mise en pratique, car on voit assez vite qu'elle est en général irréalisable. En effet, on remarquera d'abord que si la batterie peut être chargée avec la lenteur voulue (i 2 à
15 heures), il faudra par contre que sa décharge s'opère en 2 heures environ (le temps de la pointe) et sous un régime très variable, d'où destruction rapide des éléments. De plus on voit que le rendement rotal de cette double transformation atteindra à peine 50 pour roo de l'énergie fournie par les alternateurs $\left({ }^{\star}\right)$. Enfin que coûterait l'entretien d'une telle batterie, fonctionnant dans les conditions indiquées? C'est un point sur lequel il est difficile d'ètre fixé a priori.

On ne peut songer à l'emploi de ce procédé que dans le cas de pointes de faible hauteur et de longue durée. Alors on pourrait sans doute simplifier l'installation ef améliorer son rendement par l'adoption des dispositifs suivants : les alternateurs de la station génératrice, au lieu d'être de l'un des types ordinairement employés, seraient du système dit " à double courant» dont on a déjà fait avec succès plusieurs applications. En même temps qu'ils fourniraient du courant alternatif au réseau, ils enverraient du courant continu dans la batterie d'accumulateurs, lequel courant restitué aux machines dans la période de charge maximum serait par elles converti en alternatif et lancé dans la ligne de transport avec leur courant direct. On éviterait ainsi l'achat d'une commutatrice et bien des inconvénients qu'elle entraîne.

Malgré sa complication ce dispositif serait tout de même à employer quand on ne pourra pas mettre en cuvre l'un des deux moyens dont il me reste à parler.

Le plus pratique de tous - lorsque la disposition du terrain le permet - est sans contredit l'aménagement, sur le canal d'amenée de la chute, d'un réservoir compensateur assez grand pour emmagasiner dans les heures de faible charge le cube nécessaire au passage de la pointe. Là encore il ne s'agit, bien entendu, que d'un ouvrage destiné à fournir pendant quelques heures de l'annèe ces kilowatts qui rendent disponibles pour l'électrochimie les I ooo chevaux de la partie supérieure du diagramme de charge; il n'est pas question d'établir un réservoir devant faire la régularisation proprement dite du débit de la rivière. Dès lors le bassin dont il s'agit n'a pas besoin d'avoir, dans le cas qui nous occupe, de très grandes dimensions. En effet, étant reconnu que les basses chutes ne serviront. pas en général à faire de l'électrochimie et comme il n'est pas question d'envisager la régularisation permanente du débit par un lac naturel ou artificiel, nous avons à considérer, seulement pour les moyennes et hautes chutes, la capacité des réservoirs devant suffire à passer des pointes de I ooo chevaux pendant deux heures.

Il est facile d'établir la formule générale qui exprime. pour une puissance donnée le cube du réservoir en fonction de la hauteur de chute et du temps.

Pour simplifier, remplaçons l'aire de la pointe à passer par celle d'un rectangle équivalent ayant pour hauteur la dimension maximum de la pointe, soit $C$ chevaux, et pour largeui un temps moyen $T$ secondes. Le travail total en chevaux-secondes, à effectuer pour la passer sera T.C. En admettant que ce travail soit restitué à l'installation par

$\begin{array}{cccc}\text { (*) Ire phase : charge } & \text { (Accumulateurs) } & 2^{2} \text { phase : décharge } & \text { Rendement tolal } \\ \text { Commut. }=0,90 & =0,55 & \text { Commut. }=0,95 & 0,47\end{array}$


un groupe électrogène avec un rendement de $7^{5}$ pour 100 , le débit à la seconde $q$, exprimé en mètres cubes, qui fournit ce travailsous la charge dyndmique $\left.(H-\lambda){ }^{*}\right)$ se tire de l'expression:

$$
\frac{1000 q(H-\lambda)}{7^{5}} \times \frac{7^{5}}{100}=C, \quad q=\frac{C}{10(H-\lambda)}
$$

Ce qui donne pour la capacité cherchée du réservoir:

$$
Q=T q=\frac{C \cdot T}{\mathrm{Io}(H-\lambda)}
$$

Nous avons exprimé par des courbes (fig. 5) les volumes qu'il faut donner aux réservoirs, suivant les hauteurs de chute, pour réaliser pendant une heure $250,500,1000$ et 2000 chevaux. En multipliant par 2 ou par 3 les volumes portés en ordonnées on obtient les capacités des réservoirs capables de fournir ses puissances pendant des pointes de 2 ou 3 heures. Elles montrent tout de suite que, dans le cas d'une chute de 100 mètres par exemple, il faudra pour passer une pointe de 1000 HP pendant 2 heures un réservoir de $2 \times 3600=7200^{\mathrm{m}^{3}}$. Si, au contraire, la chute est de 300 mètres il ne faudra plus pour passer la même pointe que $2 \times$ I $200=$ $2400^{\mathrm{m} 3}$. Une pointe de 500 chevaux pendant 3 heures exigera dans le cas d'une chute de 100 mètres un bassin de $3 \times 1800=5400^{\mathrm{m}^{3}}$ et une pointe de 2000 chevaux pendant le même temps, exigera le même cube dans le cas d'une chute de 400 mètres. Etc...

Ces réservoirs n'ont donc pas besoin d'être bien grands et leur établissement doit, en maintes circonstances, ressortir à des prix qui le justifient amplement. Ils peuvent être obtenus soit par surélévation du barrage de prise d'eau, soit par élargissement du canal d'amenée, soit de toute autre manière plus commode, indiquée par la disposition du terrain. Une objection toutefois s'oppose à leur création. Quand il s'agit de chutes inférieures à 100 mètres et de pointes hautes et longues à passer, on est alors conduit à augmenter considérablement les capacités $\left({ }^{\star \star}\right)$. Si un industriel

(*) La pression statique étant représentée par $H$, et la perte de charge sur la hauteur $H$ par $\lambda$, la pression dynamique ou hauteur de chute effective, s'exprime par $(H-\lambda)$. expression déjà adoptée dans de précédents calculs et que nous continuerons à employer.

(*) Par exemple, dans le cas d'une chute où la pression dynamique serait de 60 mètres, le réservoir, pour passer une pointe de s ooo $\mathrm{HP}$ pendant 2 heures, devrait avoir une capacité de $12000 \mathrm{~m}^{3}$. A súpposer qu'on forme ce réservoir en barrant le lit de la rivière par un barrage large de jo mètres à la crête et haut de 6 mètres suivant la ligne du talweg, on peut considérer le volume d'eau emmagasinée comme étant une pyramide triangulaire dont la base est le parement amont de l'ouvrage et la hauteur l'étendue $x$ sur laquelle on relève le plan d'eau. On aura dans ce cas:

$$
\frac{1}{3}\left(30 \times \frac{8}{2}\right) x=12000 \text { d'où } x=400 \text { mètres au moins }
$$

Et il faudra prévoir un cube plus considérable encore pour tenir compte de l'encombrement des apports, si toutefois on peut y remédier partiellement. est seul sur la rivière, il peut aux heures de faible charge s'approvisionner d'eau pour le moment de la pointe sans gêner personne; mais supposons au-dessous de lui, sur la même rivière, un, deux ou trois industriels utilisant la même eau à des travaux différents; ils viendront dire au premier: nous avons besoin de tout le débit pendant que vous remplissez votre réservoir. D'où complications et chicanes probables. Or cette situation des usines échelonnées sur les rivières est, à n'en pas douter, celle de l'avenir. Comment s'entendront-elles lorsqu'elles voudront faire non seulement le passage de la pointe du diagramme des chevaux permanents, mais encore régulariser leur débit pour parer aux à-coups des périndes de sécheresse ?... C'est une question à laquelle j'aime mieux ne pas répondre.

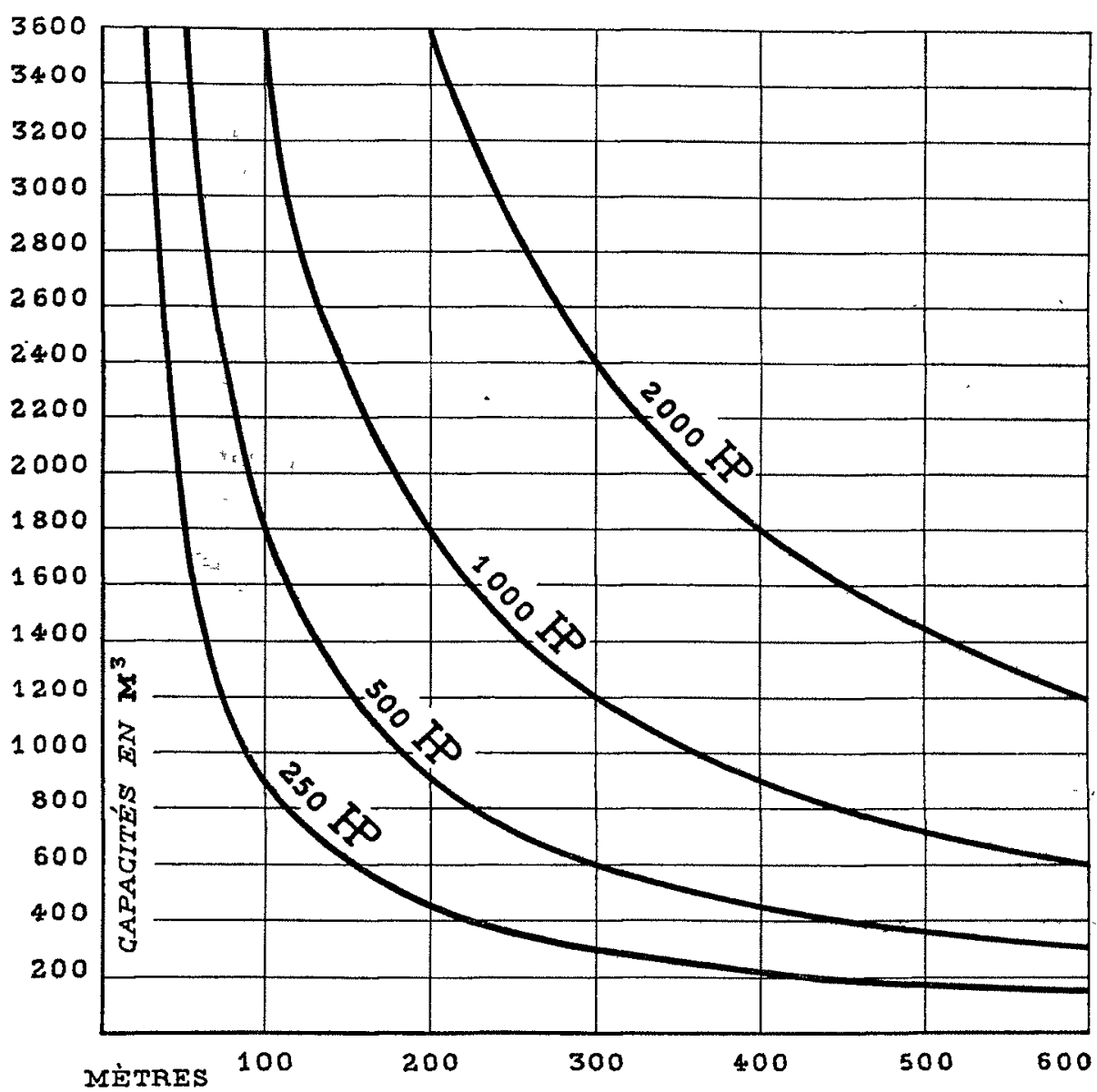

Fig. 5. - Courbes figuratives de la capacité, en fonction de la hauteur de chute, que doit avoir un réservolr pour passer des pointes de $250,500,1000$ ou 2000 que dolt avoir un reservolr
chevaux pendant une heure

Nouveau moyen de passer la pointe. - Les procédés précédents sont bien connus et, dans un certain nombre d'installations, déjà employés pour répondre plus ou moins complètement au but indiqué. Mais quand les circonstances rendent leur adoption trop difficultueuse, il peut être intéressant d'étudier la mise en œuvre d'un autre procédé qui est souvent venu à l'esprit de beaucoup d'inventeurs, mais auquel on ne s'est jamais arrêté, sans doute parce que la démonstration du paradoxe hydraulique dont it dérive n'a jamíais été faite à l'aide d'arguments précis. Il consiste, en effet, à utiliser le travail d'une chute pour remonter de l'eau dans un réservoir d'où on la laissera retomber sur les turbines !... A première vue, cette proposition semble ne pouvoir émaner que d'un ingénieur dont le cerveau finit de s'user à Charenton. 
Elle n'est cependant pas aussi naïve qu'elle en a l'air. Reportons-nous aux graphiques de la figure 5 . Alors que dans le cas d'une chute de roo mètres il faut, pour passer une pointe de 1 ooo HP pendant 2 heures, un réservoir de $7.200^{m^{3}}$, il n'est plus nécessaire pour passer la même pointe, dans le cas d'une chute de 600 mètres, que d'avoir un bassin de $120 \mathrm{~m}^{3}$. On peut alors très rationnellement employer une partie du travail de la chute de 100 mètres à refouler cette faible quantité d'eau à 600 mètres. Si l'on met, par exemple, 12 heures pour la refouler on n'absorbera pendant ce temps. que 400 chevaux environ (tous rendement compris) du travail de la chute-et si la puissance de cette dernière est seulement de 2 à 3000 chevaux, on voit qu'il est avantageux d'employer ainsi ces chevaux dans les moments où ils n'auraient presque rien à faire. Il en résulte immédiatement que le procédé-du réservoir, souvent difficile à appliquer faute d'espace, devient plus commode grâce à l'extrême réduction de cet ouvrage.

Le dispositif mécanique auquel on songe tout de suite comprend alors une pompe rotative ou bien encore une pompe alternative à grande vitesse, du type Riedler par exemple, placée à la chambre de mise en charge de l'usine et mue par un électromoteur,élevant l'eau dans un bassin établi à la hauteur voulue. Cette pompe refoule dans la conduite forcée rajoutée à l'installation et qui conduit l'eau du petit réservoir sur un groupe électrogène supplémentaire comprenant une turbine à haute chute. Reste maintenant à savoir s'il existe des pompes rotatives pouvant répondre aux conditions du problème: refoulement d'un débit de 200 à $300^{3}$ à l'heure à plusieurs centaines de mètres?

Ces pompes existent, témoin les gravures ci-jointes qui en montrent les principaux types et des applications couramment réalisées. Elles sont, on le sait déjà, formées de đisques à aubages' calés sur un même arbre horizontal, en plus ou moins grand nombre suivant les hauteurs de refoulement à atteindre, et tournant en général-à plû̀s-ode I 000 tours par minute.

La hauteur de refoulement n'est, dans certains types, limitée que par la résistance du métal, au dire des constructeurs; mais comme le rendement diminue au fur et à mesure qu'elle s'accroît au delà de certaines limites on est souvent conduit, lorsqu'il s'agit de vaincre des hauteurs de 4 à 500 mètres, à coupler deux pompes en série, l'une refoulant dans le tuyau d'aspiration de l'autre. On réncontre de nombreux exemples de ce genre dans les installations récentes pour l'épuisement de l'eau dans les mines. Il est cependant possible, renseignements des constructeurs en mains, d'obtenir le refoulement de $30 \mathrm{om}^{3}$ à l'heure à 400 mètres de hauteur avec une seule unité.

L'exécution pratique du disposif dont je viens de parler. est donc chose absolument faisable. On pourra par exemple employer un groupe semblable à celui représenté fig. 6 , où la pompe est conduite à très grande vitesse par un électromoteưr alternatif, ou encore tout autre groupe du genre de ceux des figures 8-9 ci-après, dans lesquels la pompe est commandée directement par le moteur électrique.

Contrairement à ce qu'on pourrait croire on n'aura pas, en général, avantage à refouler à de très grandes hauteurset ce qui suit va nous expliquer la raison de cette apparente contradiction :

Si l'on établit le réservoir très haut, sa capacité sera très faible, comme le montrent les diagrammes de la figure 5 ,et par suite son prix sera peu élevé ; mais par contre il faudra une conduite très longue, donc très coûteuse : son diamë: tre sera faible;il est vrai, mais la pression y pourra atteindre

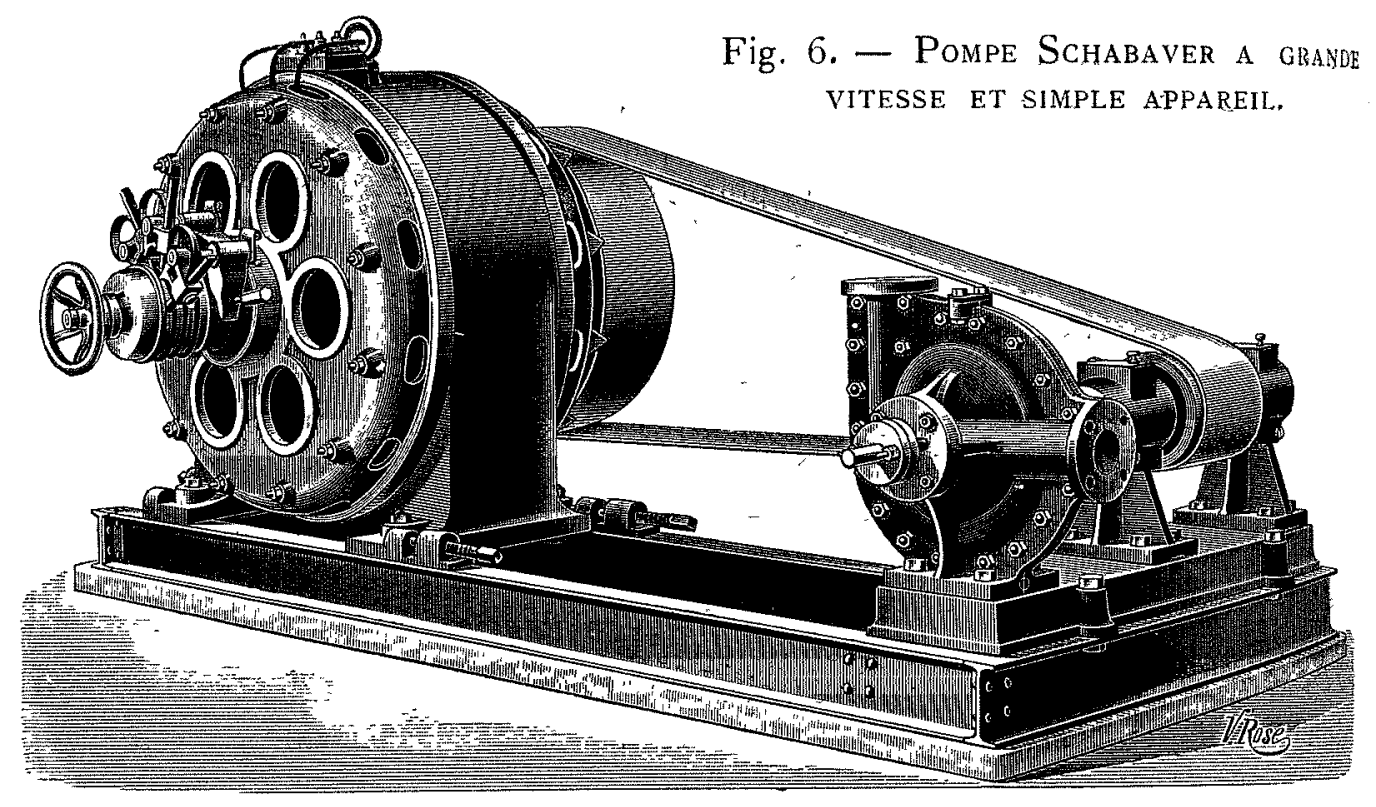

des valeurs considérables nécessitant de grandes épaisseurs. Il résulte de ces conditions que, pour une puissance donnée, il doit $y$ avoir une hauteur de refoulement qui rende minimum la somme des prix d'établissement du réservoir et de la conduite. Le calcul nous donne aisément cette hauteur.

10 Calcul du cồt du réservoir. - Nous avons vu que pour réaliser une puissance de $C$ chx pendant un temps ?' (exprimé en secondes) avec une hauteur de chute effective de $(H-\lambda)$ mètres, il fallait donner au réservoir une capa* cité-Q en mètres cubes, telle que.

$$
Q=\frac{T . C}{10(H-\lambda)}
$$

En désignant par ple prix moyen du mètre cube de résert voir, le prix de če réservoir est :

$$
\dot{P}_{\mathrm{r}}=\frac{T . C \cdot \mathrm{P}}{\mathrm{IO}(H-\lambda)}, \text { et en posant } \frac{T \cdot \dot{C} \cdot \mathrm{P}}{\mathrm{IO}}=K_{1} \text {, }
$$

il vient plus simplement : $\quad P_{\mathrm{r}}=\frac{K_{1}}{(H-\lambda)}$

$2 \circ$ Calcul du coût de la conduite. - - Si maintenant l'on admet que la conduite est en morenne inclinée d'un angle sur l'horizontale, sa longueur approximinative ẹst

$$
L=\frac{H}{\sin \varphi}=\alpha H, \text { en posant } \alpha=\frac{1}{\sin \varphi}
$$




$$
\text { D'autre part; en écrivant : } \quad \varepsilon=\frac{\lambda}{H}
$$

(c'est-dire $\varepsilon$ étant le pourcentage de la perte de charge), la formule de Darcy donne : $\left(^{*}\right)$

$$
\begin{aligned}
\frac{1}{4} D J & =\frac{1}{4} D \frac{\varepsilon H}{\alpha H}=\beta V^{2} \\
V & =\sqrt[2]{\frac{D \varepsilon}{4 \alpha \beta}}
\end{aligned}
$$

Sil'on appelle $q$ le débit (en $\mathrm{m}^{3} \mathrm{~s}$ ) de la conduite, on a :

$$
q=\frac{\pi D^{2} V}{4}=\frac{C}{10(H-\lambda)}
$$

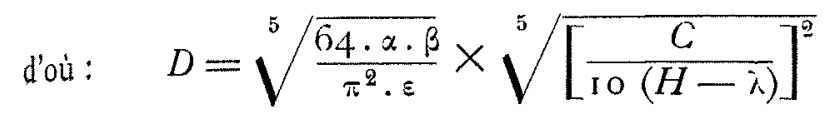

On sait que le poids (en kilogrammes) d'une conduite en tôle d'acier, que l'on fait travailler à 7 kilog. par $\mathrm{mm}^{2}$ peut se représenter approximativement par la formule:

$$
p=\alpha \cdot D^{2} \cdot H^{2} \cdot\left(^{* *}\right)
$$

En désignant par $\ldots$ le prix du kilogramme, on a pour prix de la conduite: $\quad P_{c}=\alpha . \mu . D^{2} . H^{2}$

ou bien à cause des équations (4) et (8):

$P_{\mathrm{c}}=\alpha \sqrt[5]{\left[\frac{64 \cdot \alpha \cdot \beta}{\pi^{2} \varepsilon}\right]^{2}} \times \sqrt[5]{\left[\frac{C}{10(H-\lambda)}\right]^{4}} \times\left(\frac{H-\lambda}{1-\varepsilon}\right)^{2}$ et il vient : $\quad P_{\mathrm{c}}=K_{2} \sqrt[5]{(H-\lambda)^{6}}$ en posant ;

$$
K_{2}=\frac{\alpha \mu}{(\mathrm{r}-\varepsilon)^{2}} \sqrt[5]{\left[\frac{64 \cdot \alpha \cdot \beta}{\pi^{2} \varepsilon}\right]^{2}} \times \sqrt[5]{\left(\frac{C}{10}\right)^{4}}
$$

$3^{0}$ Somme minimum du coût du réservoir et de la conduite. - D'après ce qui précède le prix total $P$ du réservoir et de la conduite sera :

$$
P=\frac{K_{1}}{H-\lambda}+K_{2} \sqrt[5]{(H-\lambda)^{6}}
$$

La valeur de $(H-\lambda)$ qui rend cette somme minimum se tire de l'équation :

$$
\frac{d P}{d(H-\lambda)}=-\frac{K_{1}}{(H-\lambda)^{2}}+\frac{6}{5} K_{2} \sqrt[5]{(H-\lambda)}=0
$$

qui

qui donne : $\quad(H-\lambda)_{\mathrm{m}}=\sqrt[11]{\left(\frac{5}{6} \frac{K_{1}}{K_{2}}\right)^{5}}$

$$
\text { et : } \quad H_{\mathrm{m}}=\frac{\mathrm{I}}{\mathrm{I}-\varepsilon} \sqrt[11]{\left(\frac{5}{6} \frac{K_{1}}{K_{\mathrm{q}}}\right)^{5}}
$$

40 Apptication numérique. - En faisant : $C=$ 1000 chevaux; $\alpha=1,5 ; \varepsilon=5$ pour $100 ; \beta=0$, 0005; et

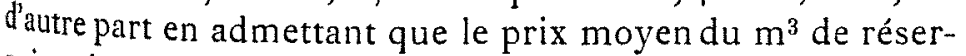
voir soit de ro francs et celui des ioo kilos de conduite de de $60 \mathrm{frs}$, on trouve :

$$
(H-\lambda)_{\mathrm{m}}=345 \text { mètres, et } H_{\mathrm{m}}=363 \mathrm{~m} 5
$$

\footnotetext{
(1) La formule de Darcy donne des sections un peu trop fortes lorsqu'il s'agit de conduites de grand diamètre, ce qui n'est pas le as ici; nous pouvons donc l'adopter pour simplifier le calcul qui sans cela serait un peu compliqué.

(1) Voir La Houille Blanche, no de mars 1904 : "Conduites forcées diamètres variables "), formules (3) et (8).
}

Ce qui donne respectivement pour les prix du réservoir et de la conduite:

$$
P_{\mathrm{r}}=20850 \text { frs. } \quad P_{\mathrm{c}}={ }_{\mathrm{I}} 7374 \text { frs. }
$$

soit un prix total 38224 frs.

La figure 7 représente graphiquement les variations de prix du réservoir et de la conduite en fonction des charges dynamiques, dans le cas de l'exemple numérique précédent.

La courbe RR donne, en milliers francs, les variations de prix du réservoir et la courbe $\mathrm{CC}$, celles du prix de la conduite; la courbe SS représente en chaque point la somme des ordonnées des deux précédentes, c'est-à-dıre le prix total.

Remarque. - Le rapport $\frac{K_{1}}{K_{0}}$ de la formule (10) peut se mettre sous la forme : $\frac{K_{1}}{K_{2}}=K_{3} \sqrt[5]{C}$ qui donne alors : $(H-\lambda)_{\mathrm{m}}=K \sqrt[11]{C}$. L'équation (10) mise sous cette forme montre ainsi que $(H-\lambda)_{\mathrm{m}}$ ne varie avec $C$ que dans des limites très restreintes.

De ce calcul il ressort donc ce fait - un peu déconcertant si l'on envisage seulement le passage de la pointe dans le diagramme des chevaux permanents, comme nous l'avons fait jusqu'à présent - que la hauteur de refoulement la plus économique est limitée à $363 / 365$ mètres, bien entendu dans le cas de l'exemple proposé (pointe de I ooo chevaux pendant 2 heures). Et encore, d'après la remarque précédente, on voit que cette hauteur varie peu avec la puissance à fournir au moment de la pointe, d'où cette conclusion que la limite industrielle de refoulement est aux environs de 400 mètres. Il en résulterait donc que puisque les básses chutes ne sont pas intéressantes pour l'électrochimie et ne nous amèneront pas à rechercher l'utilisation des chevaux permanents résiduels dans les diagrammes de charge des transports d'énergie, les applications du système proposé seraient fort restreintes. Particulièrement avantageux dans le cas des basses chutes lorsqu'un réservoir à la prise d'eau ou sur le canal d'amenée serait très coûteux ou impossible à créer faute de place, ledit système offre par contre peu d'avantage pour les chutes élevées : dès qu'on arrive à une hauteur de chute de 200 mètres, l'intérêt qu'on peut avoir à relever et emmagasiner une partie du débit à 400 mètres de hauteur n'apparaît plus. 11 est bien évident que dans le cas des hautes chutes mieux vaut créer le réservoir à même les ouvrages de dérivation et si on ne le peut pas, recourir à l'un des procédés précédemment indiqués.

Mais le système s'applique évidemment à toutes les chutes dont la hauteur est comprise entre o et 150 mètres, et -Farmi celles-ci bon nombre conviennent à l'électrochimie. Enfin, en dehors de cette dernière considération, il importe de remarquer que ce moyen de passer les pointes peut rendre des services aux installations utilisant des basses chutes uniquement pour des transports d'énergie. En leur permettant de passer les pointes d'extrême charge, par le seul secours de l'eau qu'elles n'utilisent pas aux moments de faible charge, il leur donne la possibilité de dériver un débit supérieur au minimum du cours d'eau, de créer un certain nombre de chevaux périodiques; car lorsque ceux-ci viendront à manquer, la pointe du diagramme de charge s'élevant au-dessus de la puissance 
correspondante au début minimum pourra être passée sans leur appoint. Autrement dit le procédé permet à ces usines de carrer leurs diagrammes de charge et de les inscrire dans le rectangle ayant pour hauteur l'ordonnéc du commencement et de la fin de la pointe. Cette ordonnée pourra précisément être égale à celle du débit minimum sur le graphique de débit du cours d'eau alimentant l'installation. D'où pour elles un accroissement très sensible de puissance constante.

Voyons maintenant les meilleures dispositions à adopter pour réaliser pratiquement le procédé qui nous occupe. L'installation de la pompe, mue par un noteur électrique à la chambre de mise en charge, offre l'inconvénient d'une surveillance difficile, nécessite un transport de force et oblige à placer dans l'usine un groupe turbine-dynamo pour récupérer le travail de la chute artificielle. Il est beaucoup plus simple de réaliser dans l'usine même un groupe formé d'un moteur-générateur qui actionnera d'un côté la pompe, et de l'autre sera actionné par la turbine réceptrice de la chute artificielle. Il prendra l'eau d'alimentation de la pompe sur le collecteur de la conduite forcée desservant l'usine, pour que cette pompe n'ait à vaincre qu'une hauteur de refoulement égale à la différence entre la hauteur du réservoir et celle de la chute mise en œuvre. On économise ainsi, sur tout autre dispositif, au moins une machine turbine ou dynamo. En effet, le même résultat pourrait encore s'obtenir : soit par une turbine à gros débit fonctionnant sous la basse chute, qui actionnerait la pompe par courroie avec multiplication de vitesse, et une turbine à faible débit sous la haute chute, accouplée directement avec une dỹnamo génératrice; soị au moyen d'un moteur électrique actionnant la pompe, comme le représentent par exemple les figures 8 et 9 , et $\mathrm{du}$ même groupe dynamo-turbine-haute chute. Ces procédés un peu plus compliqués que le premier offriraient par contre l'avantage de pouvoir faire tourner les machines de chaque groupe, celui de la pompe et celui de la turbine sous haute chute, aux vitesses qui leur conviennent le mieux. Il est plus avantageux de faire tourner la pompe à I 500 tours qu'à I 000 tours; de même pour la turbine sous haute chute, il est préférable de la faire tourner à 5 ou boo tours plutôt qu'à I ooo.

En réunissant ces deux groupes en un seul, comme je l'indique, on se heurte à la difficulté résultant de l'égalisation des vitesses de la pompe et de la turbine sous haute chute. Mais comme la machine électrique qui tantôt fonctionne en moteur et tantôt en génératrice peut être, dans le cas où l'usine produit du courant triphasé, un moteurgénérateur synchrone, on compense en partie la diminution du rendement de la pompe et de la turbine qui tournent, la première un peu trop lentement et la seconde un peu trop vite, par le relèvement du facteur de puissance de l'usine dû à l'introduction de cette machine - surexcitée - dans son circuit. Enfin nous croyons qu'il est possible, sans altérer beaucoup leurs rendements, de construire la pompe ef la turbine pour tourner à la même vitesse d'environ I ooo tours par minute, la première refoulant à 300 mètres par exemple et la seconde fonctionnant sous une chute dt 400 mètres $\left(^{*}\right)$. D’ailleurs les rendements ont ici un peu moins d'importance que dans le cas général d'une élévation d'eau. où l'on brûle du charbon, car nous allons voir qu'on aura toujours plus d'eau qu'il n'en faut : $1^{0}$ pour fournir le travail de refoulement, et $2^{\circ}$ pour remplir le réservoir, et cela sans rien absorber du volume d'eau constant qui correspond l'énergie rendue disponible à la partie supérieure du diagramme de charge, par le fait du passage de la pointe all moyen de notre système.

Groupe permutateur ou transpositeur d'énergie. Supposons, ce qui est un cas voisin de la réalité, une usine hydro-électrique de 3 ooo HP permanents, mis en cuvre

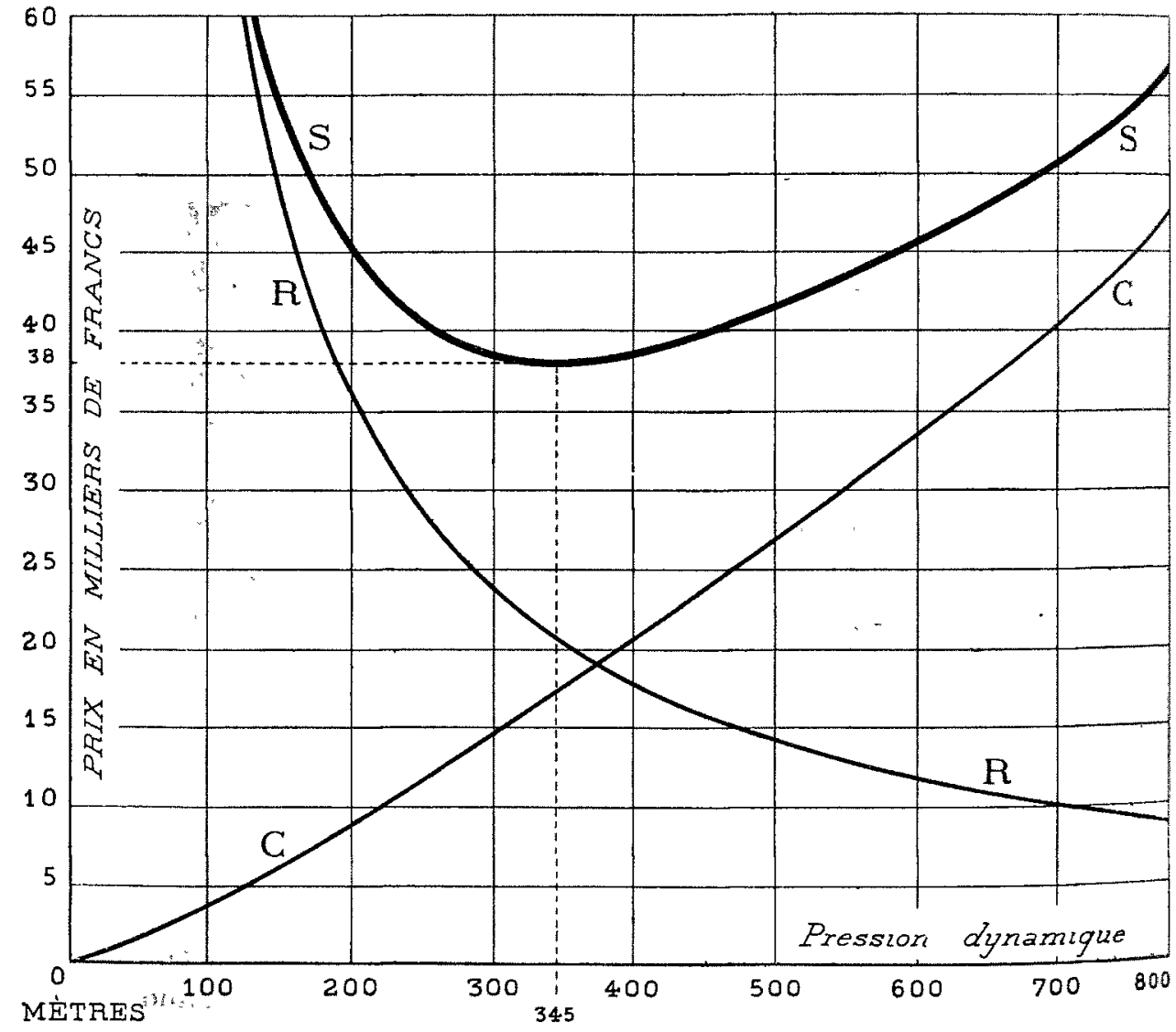

Fig. 7. - Graphiques donnant, en milliers de francs, les prix du réservoir et de la conduite, ainsi que leur somme minimum, nécessarres pour passer une pointe de I oov HP, pendant 2 heures.

par les trois groupes A, B, C, de I ooo HP chacun (fig. 10), (le groupe $C$ étant pour l'instant affecté non à l'électrochimie, mais au transport de force comme les deux autres) et alimentant un réseau dont le diagramme de charge maximum en hiver est représenté fig. I I. De $\mathrm{I}$ heure à 4 heures du matin, le groupe A fonctionne seul; de 4 heures

(t) Toutefois si cet accouplement présentait des difficultés sous le rapport des vitesses, on pourrait encore, sans beaucoup de complik cation, atteler directement la turbine à l'alternateur et commander la pompe par cet alternateur au moyen d'une courroie avec telle augmentation de vitesse qu'on le voudra. 
du matin à 4 heures du soir, les groupes $A$ et $B$ sont en marche; à partir de cette heure le groupe $\mathrm{C}$ doit entrer en fonctionnement pour passer la pointe, etc. Mais si nous adjoignons à ces trois groupes le système dont il a été question, c'est-à-dire la machine G-E composée de l'alternateur-moteur A-M, de la pompe $P$ et de la turbine $T$, nous pourrons utiliser toutes les parties pointillées $R$ du diagramme (fig. I I ), c'est-à-dire une partie des résidus d'énergie du transport de force, à relever de l'eau dans le réservoir ; au moment de la pointe cette énergie sera restituée au système en $\mathrm{G}-\mathrm{E}$ (partie la plus teintée de la fig. I I). On n'aura donc fait que transposer de l'énergie de $R, R$ en $G-E$;
Quant au rendement du groupe permutateur, voyons ce qu'll peut être. Admettons que la chute alimentant l'installation (fig. 10) soit de 63 mètres; que le rectangle équivalant à la pointe G-E (fig. I I) représente une quantité d'énergie égale à I ooo chx pendant 2 heures; qu'enfin le réservoir soit placé à 363 mètres au-dessus du niveau de l'eau dans le canal de fuite de l'usine. (C'est, comme on l'a vu, la hauteur qui donne le prix d'établissement minimum).

Le cube du réservoir devra être, d'après la formule $Q=\frac{C . T}{\text { Io }(H-\lambda)}$, de $2000 \mathrm{~m}^{3}$ en chiffres ronds. - C'est cette quantité d'eau qui, tombant de 363 mètres, pendant

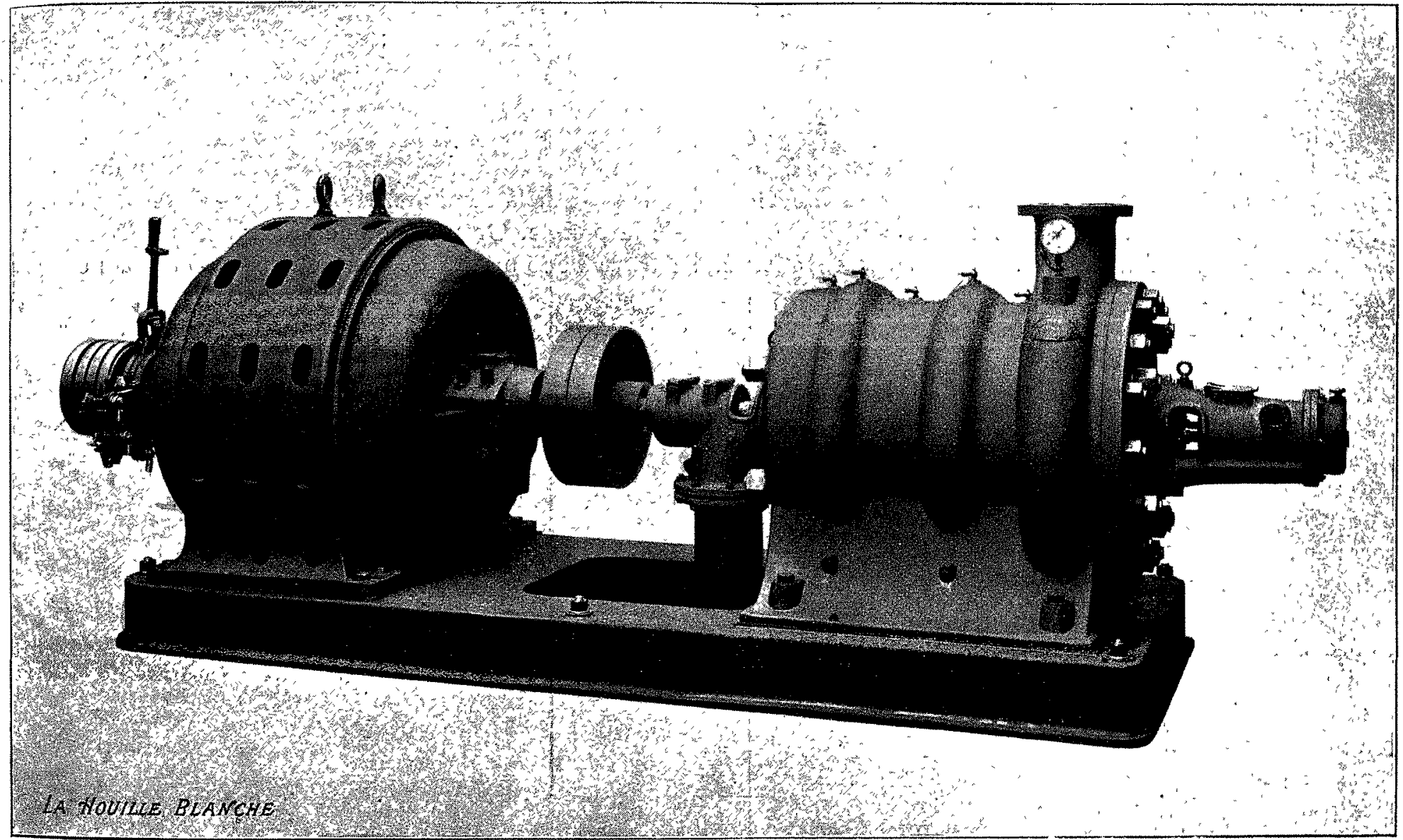

Fig. 8. - Pompe Sulzer a gros débit et grande hauteur de refioulement.

et c'est pour cela que nous donnons au système le nom de Groupe-transpositeur ou permutateur d'Énergie. Nous Voyons tout de suite sur le diagramme que les surfaces $R, R$ étant beaucoup plus grandes que celle $\mathrm{G}-\mathrm{E}$, nous pouvons admettre des rendements assez faibles pour les appareils opérant cette permutation d'énergie et par suite simplifier leur combinaison.

Grâce à l'intervention de cette machine G-E, le groupe C qui servait seulement à passer la pointe, devient inutile pourle transport de force; on peut transformer sa dynamo et par exemple l'affecter à l'électrochimie, comme le suppose linstallation schématiquement représentée fig. I1. Ce groupe aura dès lors en permanence à sa disposition toutela quantité d'énergie représentée par le rectangle teinté $C$ du diagramme fig. I I. Et ainsi, sur ses 3 ooo HP permanents, linstallation de transport de force pourra toujours en céder tooo à l'électrochimie.
2 heures, avec une perte de charge de 5 pour too et un rendement de 75 pour 100 pour le groupe récepteur turbinedynamo, produira l'énergie nécessaire au passage de la pointe. Le refoulement de cette eau peut s'opérer en Io heures. La pompe devra alors débiter $200 \mathrm{~m}^{3}$ à l'heure, soit 56 litres par seconde et le travail absorbé sera, en lui supposant un rendement de 50 pour 100 seulement :

$$
\frac{56(363-63)}{7^{5}} \times \frac{100}{50}=450 \text { chevaux. }
$$

L'alternateur-moteur fonctionnant à moitié charge (puisqu'il doit pouvoir fournir I ooo chxau moment du passage de la pointe) et étant surexcité, relève le facteur de puissance de l'usine; cela permet de considérer son rendement comme étant égal à 1 . Le rendement des groupes $\mathrm{A}$ et $\mathrm{B}$ étant pris égal à 75 pour roo, la quantité $x$ d'eau (en $\mathrm{m}^{3}$ ) nécessaire au travail de la pompe est donnée par l'expression:

$$
10 \times 63 \times x=450 \quad \text { d'où } x=\text { o m}^{3} 715 \text {. }
$$


Pour remplir le réservoir en 10 heures, il faudra donc dépenser, à la seconde, 715 lit. + 56 lit. (eau refoulée) $=77 \mathrm{I}$ litres. Ce débit, le diagramme (fig. I 1) nous permet de constater que nous pouvons le prendre, pendant tout ce temps, sur l'eau destinée à l'alimentation à pleine charge des seuls groupes A et B. En effet, les ordonnées de la courbe sunt ici proportionnelles aux débits; à l'ordonnée de 2 ooo HP correspond (sous la chute de $63 \mathrm{~m}$.) un débit de 3 I 74 litres; par conséquent à 3 I $74-77 \mathrm{I}=2403$ lit. correspond l'ordonnée de 15 r 3 chevaux. Or si l'on mène la parallèle à l'axe des abcisses passant par l'ordonnée de $1500 \mathrm{HP}$ on trouve bien que les périodes de temps d'utilisation de ce débit, qu'elle détermine par ses intersections avec la courbe de puissance, sont ensemble supériẹres à 10 heures par jour.

Quelle que soit la forme d'un diagramme de charge, on pourra toujours régler les conditions de marche d'un pareil groupe permutateur d'énergie, pour passer la pointe, en équilibrant convenablement les quantités d'énergie à transposer de part et d'autre d'une parallèle à l'axe des abcisses qui représente alors la puissance maximum des unités génératrices à faire fonctionner. Il convient en effet de remarquer que les pompes rotatives, contrairement à ce que l'on a pu croire jusqu'à ces derniers temps, peuvent fonctionner à débit variable dans d'assez larges limites, sans altération trop sensible de leur rendement. Cette propriété permettra, dans le cas par exemple du diagramme de charge figure i $\mathrm{s}$, de forcer le débit entre 1 heure et 4 heures du matin et de le restreindre vers 6 heures. Et ainsi on pourra faire fonctionner la pompe pendant plus de io heures par jour et même pendant presque tout le temps que la pointe ne se produira pas. Il serait intéressant de produire ici les courbes de rendement de ces appareils en fonction des hauteurs de refoulement et des débits, mais cette étude nous entrainerait hors du cadre de cet article et nous y reviendrons ultérieurement d'une façon détaillée s'il y a lieu.

La conduite de ce groupe apparaît comme devant être des plus simples. Lorsque le réservoir est plein, on ferme la.vanne d'alimentation de la pompe sur le collecteur des turbines - un clapet de retenue placé sur le tuyau de refoulement $p$ de la pompe empêche le retour de l'eau; puis on ouvre la vanne d'admission à la turbine sur la conduite à haute pression $t r$. Le système continue alors à tourner dans le même sens, mais l'alternateur A-M fonctionne alors en génératrice. On évite ainsi toute manœuvre d'embrayage des appareils entre eux et de couplage au tableau de distribution. Il n'y a pas d'inconvénient, vu leur faible masse, à laisser tourner à vide alternativement la, pompe et la turbine.
Parmi les objections qu'on peut faire à ce moyen de passer la pointe, il y en a deux au-devant desquelles je veux aller.

La première est celle du coût d'établissement. De prime abord il semble qu'il doive être très élevé, et tellement même qu'il faut attribuer à cette considération le fait de voir ce système a priori écarté de l'esprit lorsqu'il s'agii de résoudre ce problème de l'utilisation des résidus d'énergie. Mais nous avons vu que ce prix est rendu minimum par une certaine hauteur de refoulement variant pell avec la puissance à fournir au moment de la pointe; dans le cas de l'exemple numérique choisi plus haut (pointe de 1 ooo HP pendant 2 heures) le prix minimum ressort à 38 ooo fr. Nous avons supposé des conditions d'établissement assez favorables : réservoir à Io fr. le $\mathrm{m}^{3}$, conduite inclinée en moyenne à $4^{\circ}$ et posée à $60 \mathrm{fr}$. les 100 kilos, soit; admettons des circonstances qui en général rendent beaucoup plus chère l'application du système, et disons qu'on doit compter 50 ooo fr. pour la création du réservoir et la pose de la conduite. Il nous faut ajouter à cela le príá de la pompe. Or une pompe capable de refouler $200 \mathrm{~m}^{3} \dot{a}$ l'heure à 300 mètres de hauteur en tournant à 1000 tours

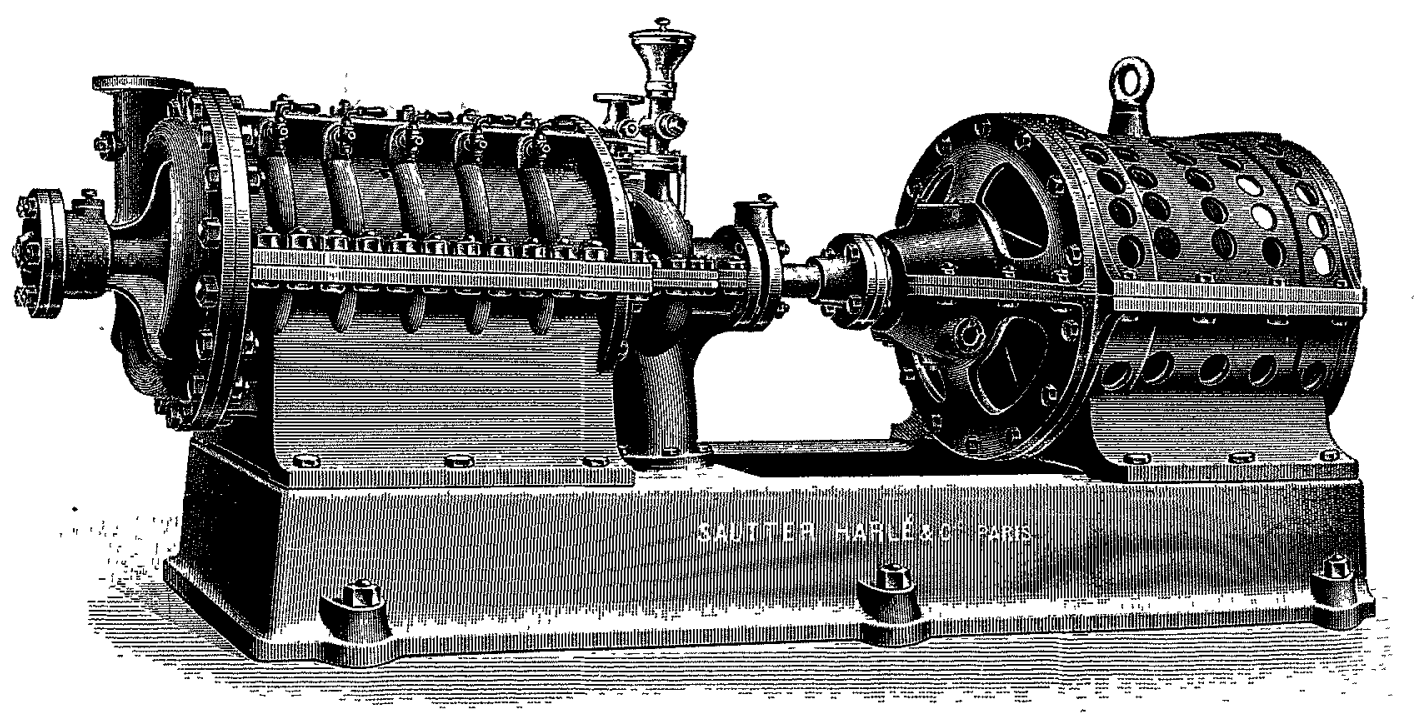

Fig. 9. - Pompe Sautter-Harlé commandėe par un alterno-moteur.

par minute coûte une vingtaine de mille francs environ et peut-être même est-ce là un maximum? La dépense totale sera done de 70 à 75000 francs pour passer une pointe de 1 ooo HP pendant 2 heures. Naturellement nous ne comptons pas la turbine sous haute chute, ni l'alternateur-moteur, puisque ces deux machines tiennent la place d'un groupe ordinaire turbine-dynamo servant à passer la pointe et qui alors peut être affecté à un autre usage (électrochimie par exemple), ou conservé comme groupe de secours si l'usine n'en possédait pas.

Le procédé n'est pas partout applicable, mais en com* parant son prix d'installation à celui des autres moyens de passer la pointe, lequel peut comme on l'a vu s'élever à 167000 fr. (toujours dans le cas de l'exemple indiqué), on verra que le cadre de ses applications possibles s'élargit beaucoup. Il offre sur l'emploi de la machine à vapeur :avantage de supprimer tous frais de combustible et de 
personnel, et sur celui des accumulateurs, d'éviter des dépenses d'entretien difficiles à estimer. On peut mème se demander si, à égalité de prix avec le système du réservoîr compensateur sur la moyenne chute, on ne doit pas donner la préférence au procédé en question qui évite radicalement tout danger de rupture du barrage par suite de crues, d'où simplification des formalités administratives pour l'établissement de l'ouvrage..., ce qui est bien aussi à considérer par le temps qui court! C'est donc de tous les systèmes, qu'un tunnel et ne doit pas en général coùter plus de 15 à $16 \mathrm{fr}$. le $\mathrm{m}^{3}$, compris tous les revêtements nécessaires sur les parois mouillées $\left(^{\star}\right)$. Cela porterait le prix du réservoir à 30 ou 32 ooo fr. au grand maximum, et avec ce prix nous sommes encore dans les limites d'application rationnelle. Enfin, comme c'est surtout pendant la nuit que le réservoir doit se remplir, on peut de plus, pour empêcher le gel, agiter l'eau en faisant déboucher le tuyau de refoulcment dans une petite galerie circulaire surélevée au-dessus du

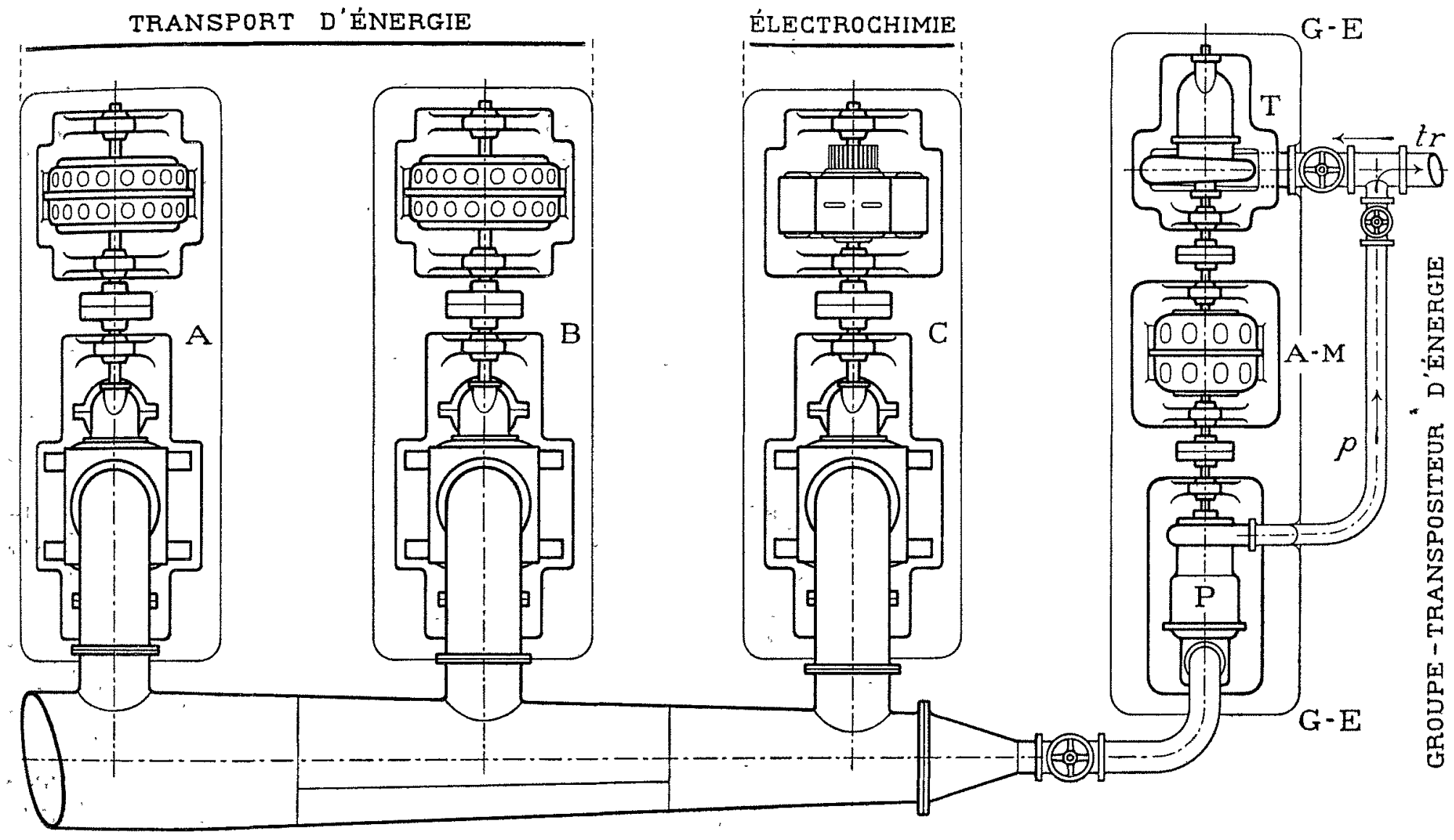

Fig. 10. - Plan schématique d'une usine avec groupe: pompe-(moteur-générateur)-turbine.

sauf l'emploi du réservoir directement placé sur la haute chute, celui qui présente le moins de frais de marche normale et d'entretien.

Là deuxième objection a précisément trait au fonctionnement normal du systeme. Quand va-t-on s'en servir? En hiver surtout, au moment où dans une usine à la fois de transport de force et d'électrochimie par exemple, les chevaux périodiques venant à manquer, il faudra prendre sur les chevaux permanents pour assurer le service des appareils de fabrication, et cela sans déranger le service public dont les diagrammes offrent, précisément à cette époque, les pointes maxima. Or, supposons l'usine établie à 6 ou 700 mètres d'altitude seulement; si l'on crée un réservoir à 300 mètres plus haut ne sera-t-il pas extrêmement exposé à se remplir de glace par les froids de - $10^{0}$ à - I 50 , assez fréquents à l'altitude de I 000 mètres? On peut éviter cet inconvénient, qui est à mon avis le plus grave, en creusant le réservoir complètement dans le rocher; une excavation de $2000^{3}$ en forme de poche ayant je suppose 50 mètres de longueur, to ou $12^{\mathrm{m}}$ de largeur et 4 ou $5 \mathrm{~m}$ de hauteur est moins difficile à faire niveau supérieur et d'ou l'eau tomberait en cascade agitant la surface pendant toute la durée du remplissage. La vidange étant très rapide, l'eau ne se congèlera pas pendant la descente. D'autre part l'eau n'étant jamais immobile dans la conduite forcée, la formation de la glace n'y est pas à craindre. Il va de soi que si le système doit fonctionner à une époque et dans une région où on n'ait pas à tenir compte de l'influence du froid, le réservoir pourra être simplement formé par appropriation d'un pli du terrain sur l'un des flancs de la gorge au fond de laquelle coule la rivière à aménager.

(*) On voit en tous cas combien ces dımensions sont différentes de celles du réservoir qu'il faudrait construire sur le canal d'amenéc de la chute de 60 mètres pour passer la même pointe. (Note de la page 149 ).

Nous avons calculé que ce réservoir doit contenir $12000 \mathrm{~m}^{3}$; pour tenir compte de l'encombrement des apports du torrent, inévitables au moins partiellement, il faut compter $15000^{113^{3}}$ au minimum. En estimant à $5 \mathrm{fr}$. le prix moyen $\mathrm{du}^{3}$, son établissement ressortirait à 75 ooo trancs; soit à un prix supérieur à celui du système proposé en supposant ce dernier établi dans des conditions défavorables. 
Je borne là l'exposé des conceptions qu'on pourrait à mon avis étudier pour faire en sorte d'arriver à une solution pratique de cette question grosse de conséquences économiques : l'utilisation des résidus d'énergie.

D'aucuns pourront peut-être trouver un peu prématurée l'étude de ces questions parce que, comme je l'ai dit plus haut, les circonstances économiques desquelles dépend la solution du problème posé ne sont point encore nées. Mais à ceux-là je réponds qu'il ne tient qu'à notre prévision de l'avenir de hâter l'ćvolution des faits générateurs de ces circonstances. L'industrie de la houille blanche a grandi d'une façon pour ainsi dire anormale en vingt ans; les chutes se sont installées comme par enchantement sous l'empire des prodiges de l'Electricité; on est allé au plus vite pour faire ici du transport de force et là de l'électro-

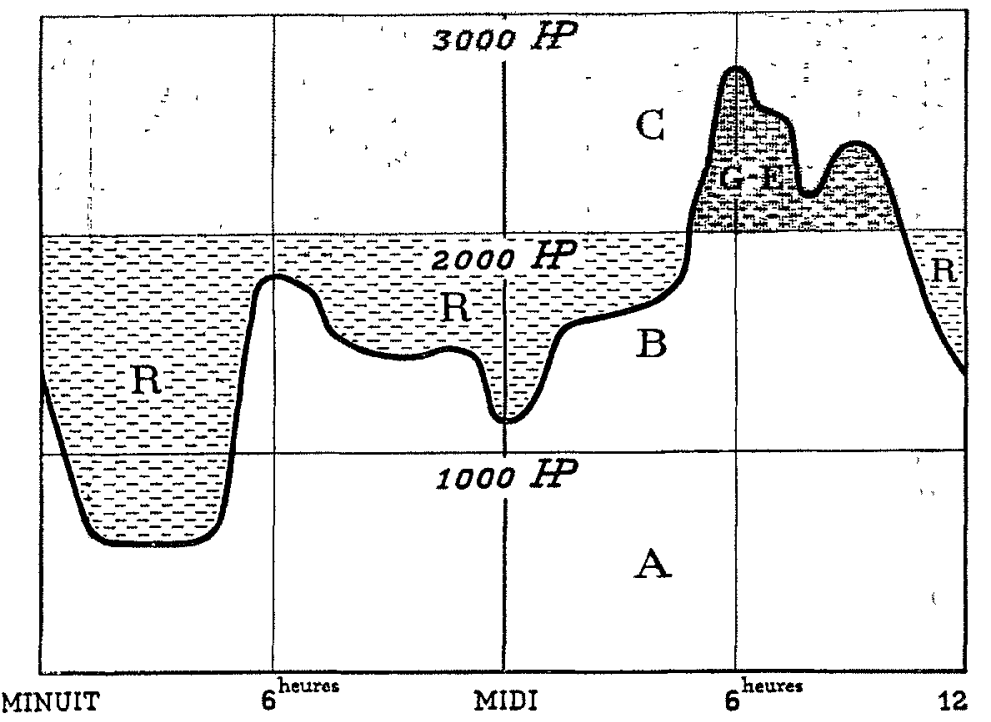

Fig. I I. - Diagramme de charge jouınalière montrant l'utilısation des chevaux permanents résiduels.

chimie; avant tout, on s'est préoccupé de capter les chutes les plus faciles à approprier et de résoudre les difficultés techniques les plus pressantes. Chacun, dans la lutte pour la meilleure place, n'a considéré que son intérêt personnel et immédiat. Il en est sans doute résulté beaucoup de progrès, mais avec pas mal de désordre. Nul plan, même hâtivement conçu, ne semble avoir été suivi dans cet essor gigantesque de la plus moderne des industries. Mais au point où nous en sommes sur cette route du progrès, n'estil pas bon de chercher à jalonner sả prolongation? Des faits acquis, résultats heureux et déboires, en apparencé pêlemêle, ne se dégage-t-il pas une leçon d'économie politique, dont on ne saurait méconnaître la portée? Quand on voit le transport de force se perfectionner sans cesse par le seul fait de la concurrence que se livrent entre eux les constructeurs pour se devancer les uns les autres, et l'électrochimie rester stationnaire, pour les diverses raisons déjà longuement énumérées en d'autres articles, n'y a-t-il pas lieu de se demander ce qu'il faut faire pour que chacune de ces deux formes essentielles de l'utilisation de nos forces naturelles, la plus belle part de notre richesse nationale, fasse rendre à ces forces leur maximum de profit?

Loin de moi la pensée d'avoir en tout cela seulement donné un coup de pioche dans le filon de la houille blanche; je voudrais simplement croire que j'ai pu faire réfléchir le mineur sur le chantier - et surtuut le législateur, de qui la réalisation des projets d'avenir du premier dépend. Car, $a^{a}$ mon sens, il ne peut être de bonne législation en cette matière si elle n'est pas fondée sur l'économie politique plutôt que réédifiée sur les anciennes bases de l'édifice juridique devenu trop étroit.

$$
* \text { * }
$$

En résumé, j’estime que lorsque dans l'aménagement d'une chute d'eau l'on veut viser la meilleure utilisalion, il faut faire deux parts de sa puissance industrielle. La première formée des " chevaux permanents " doit se calculer sur le débit minimum, c'est-à-dire sur celui au-dessous duquel le cours d'eau ne descend jamais en dix années consécutives; la deuxième comprenant les " chevaux périodiques " doit se calculer en fonction du prix de revient annuel de ces chevaux sur l'arbre des turbines : le prix du cheval périodique est donné par le rapport entre les frais à faire pour rendre l"installation apte à l'utilisation d'un certain volume d'eau en sus du débit minimum et le nombre de chevaux donné pendant 8 mois, en année moyenne, par ce volume. On pourrait appeler la somme de ces deux débits, le débit industriel.

Ces deux parts de la puissance étant faites, le meilleur rendement que l'on puisse tirer de l'installation est : $1^{0}$ de vendre cher aux services qui exigent une fourniture régulière les chevaux coûtant cher, c'est-à-dire d'affecter les chevaux permanents au transport d'énergie, et $2^{\circ}$ de vendre bon marché à l'industrie qui s'accommode de variations de puissance les chevaux coûtant peu. Et si l'on veut bien réfléchir aux considérations précédemment développées on se rendra facilement compte que cette combinaison est la plus profitable non seulement aux industriels mais encore à l'intérêt général.

En utilisant les résidus d'énergịe des chevaux perma* nents on pourra toujours assurer à l'électrochimie un large minimum de puissance constante lorsque les chevaux périodiques viendront à manquer. - En sorte que je maintiens et répète : la solution du problème de la meilleure utilisation des chutes tient dans cette formule a Transport de force et Electrochimie D.

\section{E.-F. Côte.}

\section{FIXATION DE L'AZOTE ATMOSPHÉRIQUE}

Pour compléter les divers articles qui ont déjà paru dans $L a$ Houille Blanche, sur cette importante question, nous reproduisons ci-apres la communication de M. E. C HUARD, professeur à l'Ecole d'Ingénieurs de Lausanne, telle que la donne l'excellente Reme Générale de Chimie pure et appliquée.

Parmi les problèmes qui sollicitent le plus vivement, à l'heure actuelle, l'attention des chimistes, celui de la fixation de l'azote élémentaire de l'air occupe une place à part, en ce qu'il intéresse à la fors la chimie pure, la chimie indus. trielle et la chimie agricole.

La chimie pure, en ce que les recherches faites dans celte direction ont éclairé d'un jour nouveau un domaine encore forl obscur, celui de la chimie des hautes températures et des modifications de l'affinité qui se manifestent dans ces conditions.

La chimie industrielle, à cause de l'importance des combinaisons nitrées; ces combinaisons, dont dépend en partict. 